\title{
Digital Control of Free Floating Space Robot Manipulators Using Transpose of Generalized Jacobian Matrix
}

\author{
Shinichi Sagara ${ }^{1}$ and Yuichiro Taira ${ }^{2}$ \\ ${ }^{1}$ Kyushu Institute of Technology \\ ${ }^{2}$ National Fisheries University \\ Japan
}

\section{Introduction}

Space robots having manipulators are expected to work in future space missions $(\mathrm{Xu} \&$ Kanade, 1993). Since it is difficult to supply fuel to the robots equipped with rocket motors during manipulation, control methods for free-floating space robots consisting of a base and a manipulator have been proposed (Dubowsky \& Papadopulos, 1993; Masutani et al., 1989a;b; Sagara et al., 1998a;b; Shin et al., 1995; Umetani \& Yoshida, 1989; Yamamoto et al., 1995). Most of them use the inverse of the Generalized Jacobian Matrix (GJM) which is a coefficient matrix between the velocity of the end-effector of the manipulator and the manipulator's joint velocity (Umetani \& Yoshida, 1989). Therefore, in a case that the robot manipulator gets into a singular configuration, the inverse of the GJM does not exist and the manipulator is out of control. For this problem, a continuous-time control method using the transpose of the GJM has been proposed for manipulators equipped with joint torque controllers (Masutani et al., 1989a;b).

In practical systems digital computers are utilized for controllers. So, we have proposed a discrete-time control method using the transpose of the GJM (Taira et al, 2001). The control method using the transpose of the GJM uses position and orientation errors between the desired and actual values of the end-effector. Namely, the control method belongs to a class of constant value control such as PID control. Therefore, the value of the errors depends on the desired linear and angular velocities of the end-effector based on the desired trajectory. To obtain higher control performance we have proposed a digital trajectory tracking control method that has variable feedback gains depending on the desired linear and angular velocities of the end-effector (Sagara \& Taira, 2007). Moreover, we have also proposed the control method for manipulators with velocity type joint controllers (Sagara \& Taira, 2008b).

In addition, it is considered that many tasks will be achieved by cooperative motions of several space robots in future space missions. We have studied control problems for realizing cooperative manipulations, and reported that a system consisting of space robots with manipulators and a floating object can be treated as a kind of distributed system (Katoh et al., 1997; Sagara et al., 1998b). Using the distributed system representation, each robot consisting of the distributed system can be designed by the control system individually, and we have reported a cooperative manipulation of a floating object by some space robots with the control methods using the transpose of the GJM (Sagara \& Taira, 2008a; 2009). 
In this chapter, our proposed control methods for space robot manipulators using the transpose of the GJM are described and the computer simulations are performed. First, for manipulators equipped with joint torque controllers we explain about a basic control method using constant feedback gains with the proof of stability. Next, to obtain higher control performance, we introduce a trajectory tracking control method with variable feedback gains for both torque and velocity joint inputs. Moreover, we address a cooperative manipulation of a floating object by some space robots with the control methods using the transpose of the GJM. Simulations where manipulators get into a singular configuration are also performed for the cooperative manipulation.

\section{Modelling of space robot}

We consider a free-floating space robot manipulator, as shown in Figure 1. It has an uncontrolled base and an $n$-DOF manipulator with revolute joints. Let link 0 denote a base main body, link $i(i=1, \cdots, n)$ the $i$-th link of the manipulator and joint $j$ a joint connecting link $(i-1)$ and link $i$. The target of the end-effector of the manipulator is stationary in an inertial coordinate frame.

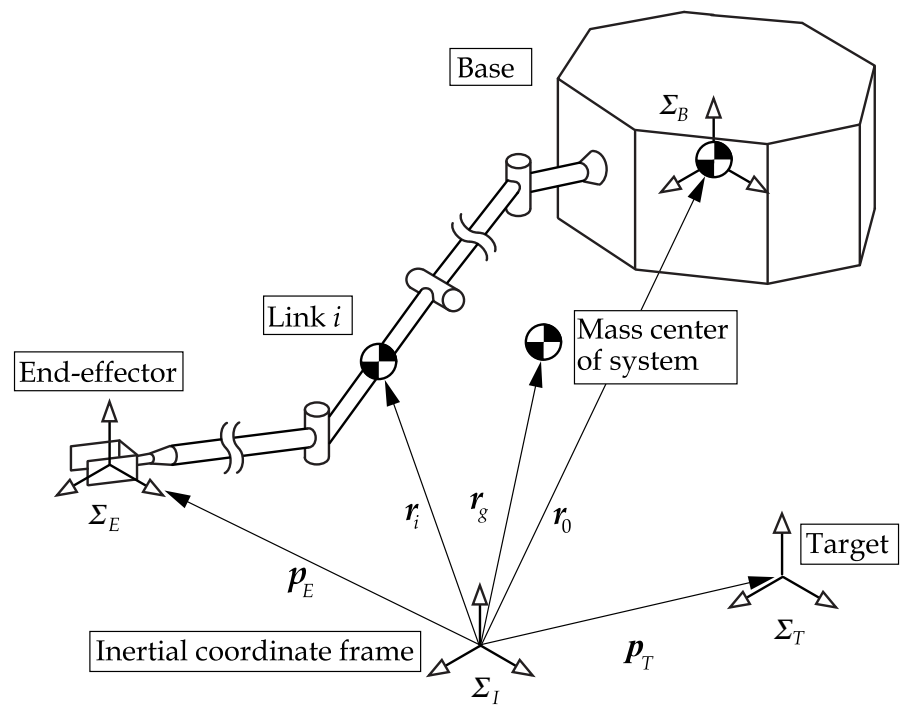

Fig. 1. Model of a space robot manipulator with an uncontrolled base

Assumptions and symbols used in this chapter are defined as follows:

\section{Assumptions}

A1) All elements of the space robot are rigid.

A2) The robot system is standing still at an initial state, i.e., the initial linear momentum and angular momentum of the space robots are zero.

A3) No external force acts on the robot system.

A4) Positions and attitude angles of robots and an object in inertial coordinate frame can be measured. 


\section{Symbols}

$\Sigma_{I}$ : inertial coordinate frame

$\Sigma_{B}$ : base coordinate frame

$\Sigma_{E}$ : end-effector coordinate frame

$\Sigma_{T}$ : target coordinate frame

$\Sigma_{i}:$ link $i$ coordinate frame

$\boldsymbol{p}_{E}:$ position vector of origin of $\Sigma_{E}$

$\boldsymbol{p}_{T}:$ position vector of origin of $\Sigma_{T}$

$\boldsymbol{p}_{i}:$ position vector of joint $i$

$\boldsymbol{r}_{0}$ : position vector of center of mass of base

$\boldsymbol{r}_{i}$ : position vector of center of mass of link $i$

$\boldsymbol{r}_{g}:$ position vector of center of mass of system

$\boldsymbol{v}_{0}:$ linear velocity vector of origin of $\Sigma_{B}$

$\boldsymbol{v}_{E}:$ linear velocity vector of origin of $\Sigma_{E}$

$\boldsymbol{\omega}_{0}:$ angular velocity vector of origin of $\Sigma_{B}$

$\boldsymbol{\omega}_{E}$ : angular velocity vector of origin of $\Sigma_{E}$

$\boldsymbol{k}_{i}$ : unit vector indicating joint axis direction of joint $i$

$\phi$ : joint angle vector

$\tau:$ joint torque vector

$m_{0}:$ mass of base

$m_{i}:$ mass of link $i$

$\boldsymbol{I}_{0}$ : inertia tensor of base

$\boldsymbol{I}_{i}$ : inertia tensor of link $i$

$\boldsymbol{E}$ : identity matrix

$\{\tilde{\bullet}\}$ : Tilde operator stands for a cross product such that $\tilde{\boldsymbol{r}} \boldsymbol{a}=\boldsymbol{r} \times \boldsymbol{a}$

Note that all vectors and inertia tensors are defined with respect to the inertial reference frame.

\section{1 kinematics and dynamics in continuous-time domain}

In this subsection, kinematics and dynamics of the robot shown in Figure 1 are briefly described. The derivation of the following equations are founded in reference (Umetani \& Yoshida, 1989).

First, a kinematic equation of the robot is described as

$$
\boldsymbol{\nu}(t)=\left[\begin{array}{l}
\boldsymbol{v}_{E}(t) \\
\boldsymbol{\omega}_{E}(t)
\end{array}\right]=\boldsymbol{J}_{S}\left[\begin{array}{l}
\boldsymbol{v}_{0}(t) \\
\boldsymbol{\omega}_{0}(t)
\end{array}\right]+\boldsymbol{J}_{m} \dot{\boldsymbol{\phi}}(t)
$$

where

$$
\boldsymbol{J}_{S}=\left[\begin{array}{cc}
\boldsymbol{E} & \tilde{\boldsymbol{r}}_{0}-\tilde{\boldsymbol{p}}_{E} \\
\mathbf{0} & \boldsymbol{E}
\end{array}\right]
$$


and

$$
\boldsymbol{J}_{m}=\left[\begin{array}{cccc}
\tilde{\boldsymbol{k}}_{1}\left(\boldsymbol{p}_{E}-\boldsymbol{p}_{1}\right) & \tilde{\boldsymbol{k}}_{2}\left(\boldsymbol{p}_{E}-\boldsymbol{p}_{2}\right) & \ldots & \tilde{\boldsymbol{k}}_{n}\left(\boldsymbol{p}_{E}-\boldsymbol{p}_{n}\right) \\
\boldsymbol{k}_{1} & \boldsymbol{k}_{2} & \ldots & \boldsymbol{k}_{1}
\end{array}\right]
$$

are Jacobian matrices for a robot base motion dependent part and a manipulator dependent part, respectively.

Next, with an assumption that total momentum of the system is held to zero, a linear and an angular momentum of the system, $\boldsymbol{P}$ and $\boldsymbol{L}$, are described as follows:

$$
\left[\begin{array}{c}
\boldsymbol{P} \\
\boldsymbol{L}
\end{array}\right]=\left[\begin{array}{cc}
w \boldsymbol{E} & w\left(\tilde{\boldsymbol{r}}_{g}-\tilde{\boldsymbol{r}}_{0}\right)^{T} \\
w \tilde{\boldsymbol{r}}_{g} & \boldsymbol{I}_{\omega}
\end{array}\right]\left[\begin{array}{c}
\boldsymbol{v}_{0}(t) \\
\boldsymbol{\omega}_{0}(t)
\end{array}\right]+\left[\begin{array}{c}
\boldsymbol{J}_{T w} \\
\boldsymbol{I}_{\phi}
\end{array}\right] \dot{\boldsymbol{\phi}}(t)=\mathbf{0}
$$

where

$$
\begin{aligned}
& \boldsymbol{I}_{\omega}=\sum_{i=1}^{n}\left\{\boldsymbol{I}_{i}-m_{i} \tilde{\boldsymbol{r}}_{i}\left(\tilde{\boldsymbol{r}}_{i}-\tilde{\boldsymbol{r}}_{0}\right)\right\}+\boldsymbol{I}_{0} \\
& \boldsymbol{I}_{\phi}=\sum_{i=1}^{n}\left(\boldsymbol{I}_{i} \boldsymbol{J}_{R_{i}}+m_{i} \tilde{\boldsymbol{r}}_{i} \boldsymbol{J}_{T_{i}}\right), \\
& \boldsymbol{J}_{T w}=\sum_{i=1}^{n} m_{i} \boldsymbol{J}_{T_{i}}, \\
& \boldsymbol{J}_{T_{i}}=\left[\tilde{\boldsymbol{k}}_{1}\left(\boldsymbol{r}_{i}-\boldsymbol{p}_{1}\right), \tilde{\boldsymbol{k}}_{2}\left(\boldsymbol{r}_{i}-\boldsymbol{p}_{2}\right), \cdots, \tilde{\boldsymbol{k}}_{i}\left(\boldsymbol{r}_{i}-\boldsymbol{p}_{i}\right), \mathbf{0}, \cdots, \mathbf{0}\right], \\
& \boldsymbol{J}_{R_{i}}=\left[\boldsymbol{k}_{1}, \boldsymbol{k}_{2}, \cdots, \boldsymbol{k}_{i}, \mathbf{0}, \cdots, \mathbf{0}\right] .
\end{aligned}
$$

Furthermore, an equation of motion of the robot is

$$
\boldsymbol{H} \ddot{\boldsymbol{\phi}}(t)+\boldsymbol{C} \dot{\boldsymbol{\phi}}(t)=\boldsymbol{\tau}(t)
$$

where

$$
\begin{gathered}
\boldsymbol{H}=\boldsymbol{H}_{\phi}-\left[\begin{array}{ll}
\boldsymbol{J}_{T w}^{T} & \boldsymbol{H}_{\omega \phi}^{T}
\end{array}\right]\left[\begin{array}{cc}
w \boldsymbol{E} & w\left(\tilde{\boldsymbol{r}}_{g}-\tilde{\boldsymbol{r}}_{0}\right)^{T} \\
w\left(\tilde{\boldsymbol{r}}_{g}-\tilde{\boldsymbol{r}}_{0}\right) & \boldsymbol{H}_{\omega}
\end{array}\right]^{-1}\left[\begin{array}{c}
\boldsymbol{J}_{T w} \\
\boldsymbol{H}_{\omega \phi}
\end{array}\right], \\
\boldsymbol{C}=\frac{1}{2} \dot{\boldsymbol{H}}+\boldsymbol{S} \\
\boldsymbol{S} \dot{\boldsymbol{\phi}}=\frac{1}{2} \dot{\boldsymbol{H}} \dot{\boldsymbol{\phi}}-\frac{\partial}{\partial \boldsymbol{\phi}}\left\{\frac{1}{2} \dot{\boldsymbol{\phi}}^{T} \boldsymbol{H} \dot{\boldsymbol{\phi}}\right\} \\
\boldsymbol{H}_{\omega}=\sum_{i=1}^{n}\left\{\boldsymbol{I}_{i}+m_{i}\left(\tilde{\boldsymbol{r}}_{i}-\tilde{\boldsymbol{r}}_{0}\right)^{T}\left(\tilde{\boldsymbol{r}}_{i}-\tilde{\boldsymbol{r}}_{0}\right)\right\}+\boldsymbol{I}_{0} \\
\boldsymbol{H}_{\omega \phi}=\sum_{i=1}^{n}\left\{\boldsymbol{I}_{i} \boldsymbol{J}_{R_{i}}+m_{i}\left(\tilde{\boldsymbol{r}}_{i}-\tilde{\boldsymbol{r}}_{0}\right) \boldsymbol{J}_{T_{i}}\right\} \\
\boldsymbol{H}_{\phi}=\sum_{i=1}^{n}\left(\boldsymbol{J}_{R_{i}}^{T} \boldsymbol{I}_{i} \boldsymbol{J}_{R_{i}}+m_{i} \boldsymbol{J}_{T_{i}}^{T} \boldsymbol{J}_{T_{i}}\right) .
\end{gathered}
$$


From Equations (1) and (2), the following relationship between the end-effector velocity and the joint velocity can be obtained:

$$
\boldsymbol{\nu}(t)=\left[\begin{array}{c}
\boldsymbol{v}_{E}(t) \\
\boldsymbol{\omega}_{E}(t)
\end{array}\right]=\boldsymbol{J} \dot{\boldsymbol{\phi}}(t)=\left[\begin{array}{l}
\boldsymbol{J}_{L} \\
\boldsymbol{J}_{A}
\end{array}\right] \dot{\boldsymbol{\phi}}(t)
$$

where

$$
\begin{gathered}
\boldsymbol{J}=\overline{\boldsymbol{J}}_{m}-\overline{\boldsymbol{J}}_{s} \boldsymbol{I}_{s}^{-1} \boldsymbol{I}_{m} \\
\boldsymbol{I}_{S}=\sum_{i=0}^{n}\left\{\boldsymbol{I}_{i}-m_{i}\left(\tilde{\boldsymbol{r}}_{i}-\tilde{\boldsymbol{r}}_{g}\right)\left(\tilde{\boldsymbol{r}}_{i}-\tilde{\boldsymbol{r}}_{g}\right)\right\}, \\
\boldsymbol{I}_{m}=\boldsymbol{I}_{\phi}-\tilde{\boldsymbol{r}}_{g} \boldsymbol{J}_{T w}, \\
\overline{\boldsymbol{J}}_{m}=\boldsymbol{J}_{m}-\boldsymbol{J}_{s}\left[\begin{array}{c}
\frac{1}{w} \boldsymbol{J}_{T w} \\
\mathbf{0}
\end{array}\right] \\
\overline{\boldsymbol{J}}_{s}=\boldsymbol{J}_{s}\left[\begin{array}{c}
\tilde{\boldsymbol{r}}_{g}-\tilde{\boldsymbol{r}}_{0} \\
\boldsymbol{E}
\end{array}\right] .
\end{gathered}
$$

$\boldsymbol{J}$ is the GJM that is an extended and generalized form of manipulator Jacobian implying the reaction dynamics of free-floating systems. $\boldsymbol{J}_{L}$ and $\boldsymbol{J}_{A}$ are the GJMs of the linear and angular velocities, respectively.

\subsection{Discrete-time representation}

To design digital control systems, discrete-time representation of Equations (4) and (3) are given.

Discretizing Equation (4) by a sampling period $T$, the following discrete-time relationship between the end-effector velocity and the joint velocity can be obtained:

$$
\begin{aligned}
\boldsymbol{v}_{E}(k) & =\boldsymbol{J}_{L}(k) \dot{\boldsymbol{\phi}}(k), \\
\boldsymbol{\omega}_{E}(k) & =\boldsymbol{J}_{A}(k) \dot{\boldsymbol{\phi}}(k) .
\end{aligned}
$$

Note that the discrete-time $k T$ is abbreviated to $k$.

Similarly, the equation of motion (3) is descretized applying $\ddot{\phi}(k)$ to the backward Euler approximation:

$$
\dot{\phi}(k)=\dot{\phi}(k-1)-T \boldsymbol{H}^{-1}(k)\{\boldsymbol{C}(k) \dot{\phi}(k)-\boldsymbol{\tau}(k)\}
$$

where

$$
\begin{gathered}
\boldsymbol{C}(k)=\frac{1}{2} \dot{\boldsymbol{H}}(k)+\boldsymbol{S}(k), \\
\boldsymbol{S}(k) \dot{\boldsymbol{\phi}}(k)=\frac{1}{2} \dot{\boldsymbol{H}}(k) \dot{\boldsymbol{\phi}}(k)-\frac{\partial}{\partial \phi(k)}\left\{\frac{1}{2} \dot{\boldsymbol{\phi}}^{T}(k) \boldsymbol{H}(k) \dot{\boldsymbol{\phi}}(k)\right\} .
\end{gathered}
$$




\subsection{Position and orientation errors}

In this subsection, a position and an orientation errors, which are used as feedback signals in the control methods described below, are defined (Taira et al, 2001).

\subsubsection{Position error}

The discrete-time position error between the target and the end-effector is defined as

$$
\boldsymbol{e}_{L}(k)=\boldsymbol{p}_{T}-\boldsymbol{p}_{E}(k) .
$$

Using Equations (5) and (8), and the backward Euler approximation of $\boldsymbol{v}_{E}$, i.e.,

$$
\boldsymbol{v}_{E}(k)=\frac{1}{T}\left\{\boldsymbol{p}_{E}(k)-\boldsymbol{p}_{E}(k-1)\right\},
$$

the following difference equation of the position error is obtained:

$$
\begin{aligned}
\boldsymbol{e}_{L}(k) & =\boldsymbol{e}_{L}(k-1)-T \boldsymbol{v}_{E}(k) \\
& =\boldsymbol{e}_{L}(k-1)-T \boldsymbol{J}_{L}(k) \dot{\phi}(k) .
\end{aligned}
$$

\subsubsection{Orientation error}

In order to represent orientation of the end-effector and the target, we introduce the following rotation matrices:

$$
\begin{gathered}
\boldsymbol{A}_{E}(t)=\left[\begin{array}{lll}
\boldsymbol{n}_{E}(t) & \boldsymbol{s}_{E}(t) & \boldsymbol{a}_{E}(t)
\end{array}\right], \\
\boldsymbol{A}_{T}=\left[\begin{array}{lll}
\boldsymbol{n}_{T} & \boldsymbol{s}_{T} & \boldsymbol{a}_{T}
\end{array}\right]
\end{gathered}
$$

where $\boldsymbol{n}_{*}, \boldsymbol{s}_{*}$ and $\boldsymbol{a}_{*}(*=E, T)$ are unit vectors along the axes of $\Sigma_{*}$ with respect to $\Sigma_{I}$, and

$$
\left.\begin{array}{rl}
\dot{\boldsymbol{n}}_{E}(t) & =\tilde{\boldsymbol{\omega}}_{E}(t) \boldsymbol{n}_{E}(t)=-\tilde{\boldsymbol{n}}_{E}(t) \boldsymbol{\omega}_{E}(t) \\
\dot{\boldsymbol{s}}_{E}(t)=\tilde{\boldsymbol{\omega}}_{E}(t) \boldsymbol{s}_{E}(t)=-\tilde{\boldsymbol{s}}_{E}(t) \boldsymbol{\omega}_{E}(t) \\
\dot{\boldsymbol{a}}_{E}(t)=\tilde{\boldsymbol{\omega}}_{E}(t) \boldsymbol{a}_{E}(t)=-\tilde{\boldsymbol{a}}_{E}(t) \boldsymbol{\omega}_{E}(t)
\end{array}\right\}
$$

is satisfied.

The continuous-time orientation error between the target and the end-effector is defined as

$$
\boldsymbol{E}_{A}(t)=\left[\begin{array}{c}
\boldsymbol{n}_{T}-\boldsymbol{n}_{E}(t) \\
\boldsymbol{s}_{T}-\boldsymbol{s}_{E}(t) \\
\boldsymbol{a}_{T}-\boldsymbol{a}_{E}(t)
\end{array}\right] .
$$

Using Equations (6), (12) and (13), we have

$$
\dot{\boldsymbol{E}}_{A}(t)=-\left[\begin{array}{c}
\dot{\boldsymbol{n}}_{E}(t) \\
\dot{\boldsymbol{s}}_{E}(t) \\
\dot{\boldsymbol{a}}_{E}(t)
\end{array}\right]=\boldsymbol{E}_{X}(t) \boldsymbol{J}_{A}(t) \dot{\boldsymbol{\phi}}
$$

where

$$
\boldsymbol{E}_{X}(t)=\left[\begin{array}{c}
\tilde{\boldsymbol{n}}_{E}(t) \\
\tilde{\boldsymbol{s}}_{E}(t) \\
\tilde{\boldsymbol{a}}_{E}(t)
\end{array}\right] .
$$


Discretizing Equation (14) by the sampling period $T$, and the backward Euler approximation of $\dot{\boldsymbol{E}}_{A}$, the following difference equation of the orientation error is obtained:

$$
\boldsymbol{E}_{A}(k)=\boldsymbol{E}_{A}(k-1)+T \boldsymbol{E}_{X}(k) \boldsymbol{J}_{A}(k) \dot{\boldsymbol{\phi}}(k) .
$$

Furthermore, the discrete-time orientation error used in the control method described below is defined as

$$
\boldsymbol{e}_{A}(k)=\frac{1}{2}\left\{\tilde{\boldsymbol{n}}_{E}(k) \boldsymbol{n}_{T}+\tilde{\boldsymbol{s}}_{E}(k) \boldsymbol{s}_{T}+\tilde{\boldsymbol{a}}_{E}(k) \boldsymbol{a}_{T}\right\} .
$$

It should be noted that a relationship between $\boldsymbol{E}_{A}$ and $\boldsymbol{e}_{A}$ is given by the following equation:

Hence, if a condition

$$
\boldsymbol{e}_{A}(k)=-\frac{1}{2} \boldsymbol{E}_{X}^{T}(k) \boldsymbol{E}_{A}(k)
$$

$$
\boldsymbol{n}_{E}^{T}(k) \boldsymbol{n}_{T}+\boldsymbol{s}_{E}^{T}(k) \boldsymbol{s}_{T}+\boldsymbol{a}_{E}^{T}(k) \boldsymbol{a}_{T}>-1
$$

is satisfied, $\boldsymbol{E}_{A}(k)=\mathbf{0}$ is equivalent to $\boldsymbol{e}_{A}(k)=\mathbf{0}$ (Masutani et al., 1989b).

\section{Basic control method using transpose of GJM}

In this section, we address a basic digital control method using the transpose of the GJM (Taira et al, 2001). The control method guarantees the stability of system in discrete-time domain by using Lyapunov's direct method for difference equations.

A joint torque command is given by

$$
\boldsymbol{\tau}_{d}(k)=\boldsymbol{J}_{L}^{T}(k)\left\{k_{L}(k) \boldsymbol{e}_{L}(k)-\boldsymbol{K}_{L}(k) \boldsymbol{v}_{E}(k)\right\}+\boldsymbol{J}_{A}^{T}(k)\left\{k_{A}(k) \boldsymbol{e}_{A}(k)-\boldsymbol{K}_{A}(k) \boldsymbol{\omega}_{E}(k)\right\}
$$

where, $k_{\dagger}(\dagger=L, A)$ is a positive scalar gain and $\boldsymbol{K}_{\dagger}$ is a symmetric and positive definite gain matrix.

The following theorem establishes the stability properties of the Equation (18).

Theorem 1. Assume that the joint torque of the manipulator is identical to the joint torque command given by Equation (18), i.e., $\boldsymbol{\tau}(k) \approx \tau_{d}(k)$, and number of joints is $n=6$. In the system represented by Equations (7), (9), (15) and (18), the equilibrium state

$$
\boldsymbol{r}_{E}(k)=\boldsymbol{r}_{T}, \boldsymbol{n}_{E}(k)=\boldsymbol{n}_{T}, \boldsymbol{s}_{E}(k)=\boldsymbol{s}_{T}, \boldsymbol{a}_{E}(k)=\boldsymbol{a}_{T}, \dot{\boldsymbol{\phi}}(k)=\mathbf{0}
$$

is asymptotically stable, if the following conditions hold during manipulation:

$$
\begin{aligned}
& \operatorname{rank}\left[\boldsymbol{J}_{L}^{T}(k) \quad \boldsymbol{J}_{A}^{T}(k)\right]=6 \quad \text { (full rank), } \\
& \boldsymbol{n}_{E}^{T}(k) \boldsymbol{n}_{T}+\boldsymbol{s}_{E}^{T}(k) \boldsymbol{s}_{T}+\boldsymbol{a}_{E}^{T}(k) \boldsymbol{a}_{T}>-1 .
\end{aligned}
$$

Proof.

We choose

$$
W(k)=W_{1}(k)+W_{2}(k)+W_{3}(k)
$$


as a candidate for a Lyapunov function which is positive definite, and its first difference $\Delta W(k)$ is defined as

$$
\Delta W(k)=W(k)-W(k-1)=\sum_{i=1}^{3} \Delta W_{i}(k)
$$

where

$$
\begin{gathered}
W_{1}(k)=k_{p} \boldsymbol{e}_{L}^{T}(k) \boldsymbol{e}_{L}(k), \\
W_{2}(k)=\frac{1}{2} k_{A} \boldsymbol{E}_{A}^{T}(k) \boldsymbol{E}_{A}(k), \\
W_{3}(k)=\dot{\phi}^{T}(k) \boldsymbol{H}(k) \dot{\boldsymbol{\phi}}(k), \\
\Delta W_{i}(k)=W_{i}(k)-W_{i}(k-1)(i=1,2,3) .
\end{gathered}
$$

From Equation (9), $\Delta W_{1}(k)$ is represented as follows:

$$
\Delta W_{1}(k)=-2 k_{L} T \dot{\boldsymbol{\phi}}^{T}(k) \boldsymbol{J}_{L}^{T}(k) \boldsymbol{e}_{L}(k)-k_{L} T^{2} \dot{\boldsymbol{\phi}}^{T}(k) \boldsymbol{J}_{L}^{T}(k) \boldsymbol{J}_{L}(k) \dot{\boldsymbol{\phi}}(k) .
$$

Similarly, using Equations (15) and (17) and the property that $\boldsymbol{E}_{X}^{T}(k) \boldsymbol{E}_{X}(k)=2 \boldsymbol{E}, \Delta W_{2}(k)$ can be rewritten as

$$
\begin{aligned}
\Delta W_{2}(k) & =k_{A} T \dot{\boldsymbol{\phi}}^{T}(k) \boldsymbol{J}_{A}^{T}(k) \boldsymbol{E}_{X}^{T}(k) \boldsymbol{E}_{A}(k)-\frac{1}{2} k_{A} T^{2} \dot{\boldsymbol{\phi}}^{T}(k) \boldsymbol{J}_{A}^{T}(k) \boldsymbol{E}_{X}^{T}(k) \boldsymbol{E}_{X}(k) \boldsymbol{J}_{A}(k) \dot{\boldsymbol{\phi}}(k) \\
& =-2 k_{A} T \dot{\phi}^{T}(k) \boldsymbol{e}_{A}(k)-k_{A} T^{2} \dot{\boldsymbol{\phi}}^{T}(k) \boldsymbol{J}_{A}^{T}(k) \boldsymbol{J}_{A}(k) \dot{\boldsymbol{\phi}}(k) .
\end{aligned}
$$

For $\Delta W_{3}(k)$, assuming $\boldsymbol{H}(k) \approx \boldsymbol{H}(k-1)$ for one sampling period, i.e., $\dot{\boldsymbol{H}}(k) \approx \mathbf{0}$, and using Equation (7) and the property

$$
\dot{\phi}^{T}(k) \boldsymbol{S}(k) \dot{\phi}(k)=\mathbf{0},{ }^{\forall} k
$$

we have

$$
\Delta W_{3}(k)=2 T \dot{\boldsymbol{\phi}}^{T}(k) \boldsymbol{\tau}(k)-T^{2}\{\boldsymbol{S}(k) \dot{\boldsymbol{\phi}}(k)-\boldsymbol{\tau}(k)\}^{T} \boldsymbol{H}^{-1}(k)\{\boldsymbol{S}(k) \dot{\boldsymbol{\phi}}(k)-\boldsymbol{\tau}(k)\}
$$

Substituting $\boldsymbol{\tau}(k) \approx \boldsymbol{\tau}_{d}(k)$ and Equations (5), (6) and (18) into Equation (30) yields

$$
\begin{aligned}
\Delta W_{3}(k)= & 2 k_{L} T \dot{\boldsymbol{\phi}}^{T}(k) \boldsymbol{J}_{L}^{T}(k) \boldsymbol{e}_{L}(k)+2 k_{A} T \dot{\boldsymbol{\phi}}^{T}(k) \boldsymbol{J}_{A}^{T}(k) \boldsymbol{e}_{L}(k) \\
& -2 T \dot{\boldsymbol{\phi}}^{T}(k) \boldsymbol{J}_{L}^{T}(k) \boldsymbol{K}_{A} \boldsymbol{v}_{E}(k)-2 T \dot{\boldsymbol{\phi}}^{T}(k) \boldsymbol{J}_{A}^{T}(k) \boldsymbol{K}_{A} \boldsymbol{\omega}_{E}(k) \\
& -T^{2}\left\{\boldsymbol{S}(k) \dot{\boldsymbol{\phi}}(k)-\boldsymbol{\tau}_{d}(k)\right\}^{T} \boldsymbol{H}^{-1}(k)\left\{\boldsymbol{S}(k) \dot{\boldsymbol{\phi}}(k)-\boldsymbol{\tau}_{d}(k)\right\} \\
= & 2 k_{L} T \dot{\boldsymbol{\phi}}^{T}(k) \boldsymbol{J}_{L}^{T}(k) \boldsymbol{e}_{L}(k)+2 k_{A} T \dot{\boldsymbol{\phi}}^{T}(k) \boldsymbol{J}_{A}^{T}(k) \boldsymbol{e}_{L}(k) \\
& -2 T \dot{\boldsymbol{\phi}}^{T}(k) \boldsymbol{J}_{L}^{T}(k) \boldsymbol{K}_{A} \boldsymbol{J}_{L}(k) \boldsymbol{\phi}(k)-2 T \dot{\boldsymbol{\phi}}^{T}(k) \boldsymbol{J}_{A}^{T}(k) \boldsymbol{K}_{A} \boldsymbol{J}_{A}(k) \boldsymbol{\phi}(k) \\
& -T^{2}\left\{\boldsymbol{S}(k) \dot{\boldsymbol{\phi}}(k)-\boldsymbol{\tau}_{d}(k)\right\}^{T} \boldsymbol{H}^{-1}(k)\left\{\boldsymbol{S}(k) \dot{\boldsymbol{\phi}}(k)-\boldsymbol{\tau}_{d}(k)\right\} .
\end{aligned}
$$

From Equations (28), (29) and (31), Equation (23) can be rewritten as 


$$
\begin{aligned}
\Delta W(k)= & -k_{L} T^{2} \dot{\boldsymbol{\phi}}^{T}(k) \boldsymbol{J}_{L}^{T}(k) \boldsymbol{J}_{L}(k) \dot{\boldsymbol{\phi}}(k)-k_{A} T^{2} \dot{\boldsymbol{\phi}}^{T}(k) \boldsymbol{J}_{A}^{T}(k) \boldsymbol{J}_{A}(k) \dot{\boldsymbol{\phi}}(k) \\
& -2 T \dot{\boldsymbol{\phi}}^{T}(k) \boldsymbol{J}_{L}^{T}(k) \boldsymbol{K}_{A} \boldsymbol{J}_{L}(k) \boldsymbol{\phi}(k)-2 T \dot{\boldsymbol{\phi}}^{T}(k) \boldsymbol{J}_{A}^{T}(k) \boldsymbol{K}_{A} \boldsymbol{J}_{A}(k) \boldsymbol{\phi}(k) \\
& -T^{2}\left\{\boldsymbol{S}(k) \dot{\boldsymbol{\phi}}(k)-\boldsymbol{\tau}_{d}(k)\right\}^{T} \boldsymbol{H}^{-1}(k)\left\{\boldsymbol{S}(k) \dot{\boldsymbol{\phi}}(k)-\boldsymbol{\tau}_{d}(k)\right\} \\
= & -2 T \dot{\boldsymbol{\phi}}^{T}(k) \overline{\boldsymbol{K}}(k) \dot{\boldsymbol{\phi}}(k)-T^{2} \dot{\boldsymbol{\phi}}^{T}(k) \overline{\boldsymbol{Q}}(k) \dot{\boldsymbol{\phi}}(k) \\
& -T^{2}\left\{\boldsymbol{S}(k) \dot{\boldsymbol{\phi}}(k)-\boldsymbol{\tau}_{d}(k)\right\}^{T} \boldsymbol{H}^{-1}(k)\left\{\boldsymbol{S}(k) \dot{\boldsymbol{\phi}}(k)-\boldsymbol{\tau}_{d}(k)\right\} \\
\leq & 0
\end{aligned}
$$

where

$$
\begin{aligned}
& \overline{\boldsymbol{K}}(k)=\left[\begin{array}{ll}
\boldsymbol{J}_{L}{ }^{T}(k) & \boldsymbol{J}_{A}^{T}(k)
\end{array}\right]\left[\begin{array}{cc}
\boldsymbol{K}_{L} & \mathbf{0} \\
\mathbf{0} & \boldsymbol{K}_{A}
\end{array}\right]\left[\begin{array}{l}
\boldsymbol{J}_{L}(k) \\
\boldsymbol{J}_{A}(k)
\end{array}\right], \\
& \overline{\boldsymbol{Q}}(k)=\left[\begin{array}{ll}
\boldsymbol{J}_{L}{ }^{T}(k) & \boldsymbol{J}_{A}^{T}(k)
\end{array}\right]\left[\begin{array}{cc}
k_{L} \boldsymbol{E} & \mathbf{0} \\
\mathbf{0} & k_{A} \boldsymbol{E}
\end{array}\right]\left[\begin{array}{l}
\boldsymbol{J}_{L}(k) \\
\boldsymbol{J}_{A}(k)
\end{array}\right]
\end{aligned}
$$

and $\overline{\boldsymbol{K}}(k)$ and $\overline{\boldsymbol{Q}}(k)$ are positive definite matrices.

In Equation (32), $\Delta W(k)=0$ is satisfied if and only if $\dot{\phi}(k)=\mathbf{0}$ and $\boldsymbol{\tau}_{d}(k)=\mathbf{0}$. In addition, from Equations (5) and (6), the condition of $\dot{\phi}(k)=\mathbf{0}$ holds $\boldsymbol{v}_{E}(k)=\mathbf{0}$ and $\boldsymbol{\omega}_{E}(k)=\mathbf{0}$. Then, in the case of $\dot{\phi}(k)=\mathbf{0}$ and $\boldsymbol{\tau}_{d}(k)=\mathbf{0}$, Equation (18) becomes

$$
\left[\begin{array}{ll}
\boldsymbol{J}_{L}^{T}(k) & \boldsymbol{J}_{A}^{T}(k)
\end{array}\right]\left[\begin{array}{c}
k_{L} \boldsymbol{e}_{L}(k) \\
k_{A} \boldsymbol{e}_{A}(k)
\end{array}\right]=\mathbf{0}
$$

and this equation is equivalent to $\boldsymbol{e}_{L}(k)=\mathbf{0}$ and $\boldsymbol{e}_{A}(k)=\mathbf{0}$ under the condition (20). Furthermore, $\boldsymbol{e}_{A}(k)=\mathbf{0}$ is equivalent to $\boldsymbol{E}_{O I}=\mathbf{0}$ under the condition (21).

Therefore, since $W(k)=0$ and $\Delta W(k)=0$ are satisfied if and only if the state is (19), and the other state keeps $W(k)>0$ and $\Delta W(k)<0, W(k)$ is a Lyapunov function and the equilibrium state (19) is is asymptotically stable.

In order to validate the effectiveness of the control law (18), computer simulations were performed. Figure 2 shows the simulation model which has a 6-DOF manipulator, whose physical parameters are shown in Table 1.

To avoid excessive inputs and to improve the end-effector path from the initial point to the target, the following conditions are used:

$$
\begin{aligned}
\overline{\boldsymbol{e}}_{L}(k) & =\left\{\begin{array}{cl}
\boldsymbol{e}_{L}(k) & \left(\left\|\boldsymbol{e}_{L}(k)\right\| \leq e_{L_{\max }}\right) \\
\frac{\boldsymbol{e}_{L}(k)}{\left\|\boldsymbol{e}_{L}(k)\right\|} e_{L_{\max }} & \left(\left\|\boldsymbol{e}_{L}(k)\right\|>e_{L_{\max }}\right)^{\prime}
\end{array}\right. \\
\overline{\boldsymbol{e}}_{A}(k) & =\left\{\begin{array}{cc}
\boldsymbol{e}_{A}(k) & \left(\left\|\boldsymbol{E}_{A}(k)\right\| \leq \sqrt{2} e_{A_{\max }}\right) \\
\frac{\sqrt{2} \boldsymbol{e}_{A}(k)}{\left\|\boldsymbol{E}_{A}(k)\right\|} e_{A_{\max }} & \left(\left\|\boldsymbol{E}_{A}(k)\right\|>\sqrt{2} e_{A_{\max }}\right)^{\prime}
\end{array}\right.
\end{aligned}
$$

Simulation was carried out under the following condition. The sampling period is $T=0.01[\mathrm{~s}]$ and $\mathrm{t}$ the controller parameters are $k_{L}=150, k_{A}=150, \boldsymbol{K}_{L}=\operatorname{diag}\left[\begin{array}{ll}300 & 150600], \boldsymbol{K}_{A}=\end{array}\right.$

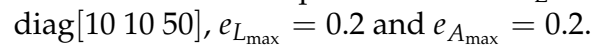




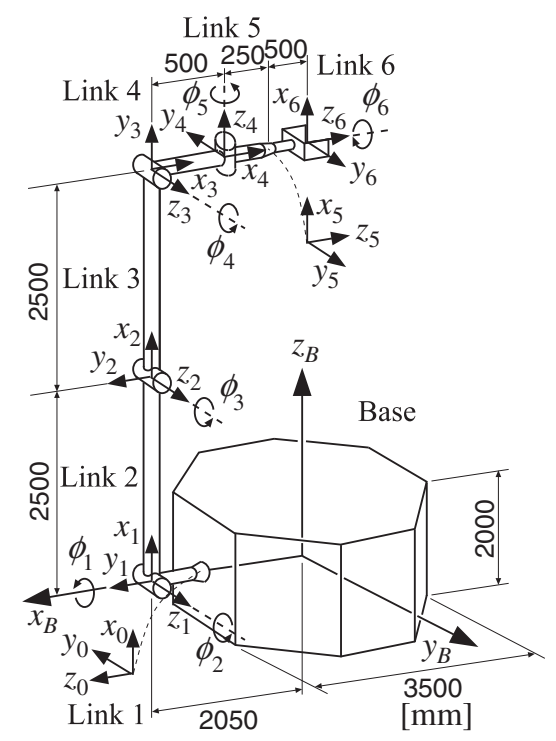

Fig. 2. Simulation model of 6-DOF space robot

\begin{tabular}{c|c|c|c|c|c}
\hline & $\begin{array}{c}\text { Length } \\
\mathrm{m}\end{array}$ & $\begin{array}{c}\text { Mass } \\
\mathrm{kg}\end{array}$ & \multicolumn{3}{|c}{$\begin{array}{c}\text { Moment of inertia } \\
\mathrm{kg} \cdot \mathrm{m}^{2}\end{array}$} \\
\cline { 3 - 6 } & & & $I_{i x}$ & $I_{i y}$ & $I_{i z}$ \\
\hline Base & 3.5 & 2000 & 1933 & 1936 & 2537 \\
\hline Link 1 & 0.25 & 5 & 0.16 & 0.06 & 0.17 \\
\hline Link 2 & 2.5 & 50 & 0.79 & 35.0 & 35.4 \\
\hline Link 3 & 2.5 & 50 & 0.79 & 35.0 & 35.4 \\
\hline Link 4 & 0.5 & 10 & 0.27 & 0.45 & 0.27 \\
\hline Link 5 & 0.25 & 5 & 0.04 & 0.06 & 0.06 \\
\hline Link 6 & 0.5 & 5 & 0.07 & 0.23 & 0.26 \\
\hline
\end{tabular}

Table 1. Physical parameters of 6-DOF space robot

Simulation result is shown in Figure 3. From this figure, we can see that the position and orientation of the end-effector are well controlled nevertheless the base is moved.

\section{Trajectory tracking control}

The basic control law (18) uses position and orientation errors between the desired and actual values of the end-effector of the manipulator. Namely, the basic control method using Equation (18) belong to a class of constant value control such as PID control. So, the basic control method can adopt a desired trajectory of the end-effector for practical purposes. The value of errors, however, depends on the desired linear and angular velocities of the end-effector based on the desired trajectory. In this section, we address digital trajectory tracking control methods using the transpose of the GJM (Sagara \& Taira, 2007; 2008b). 

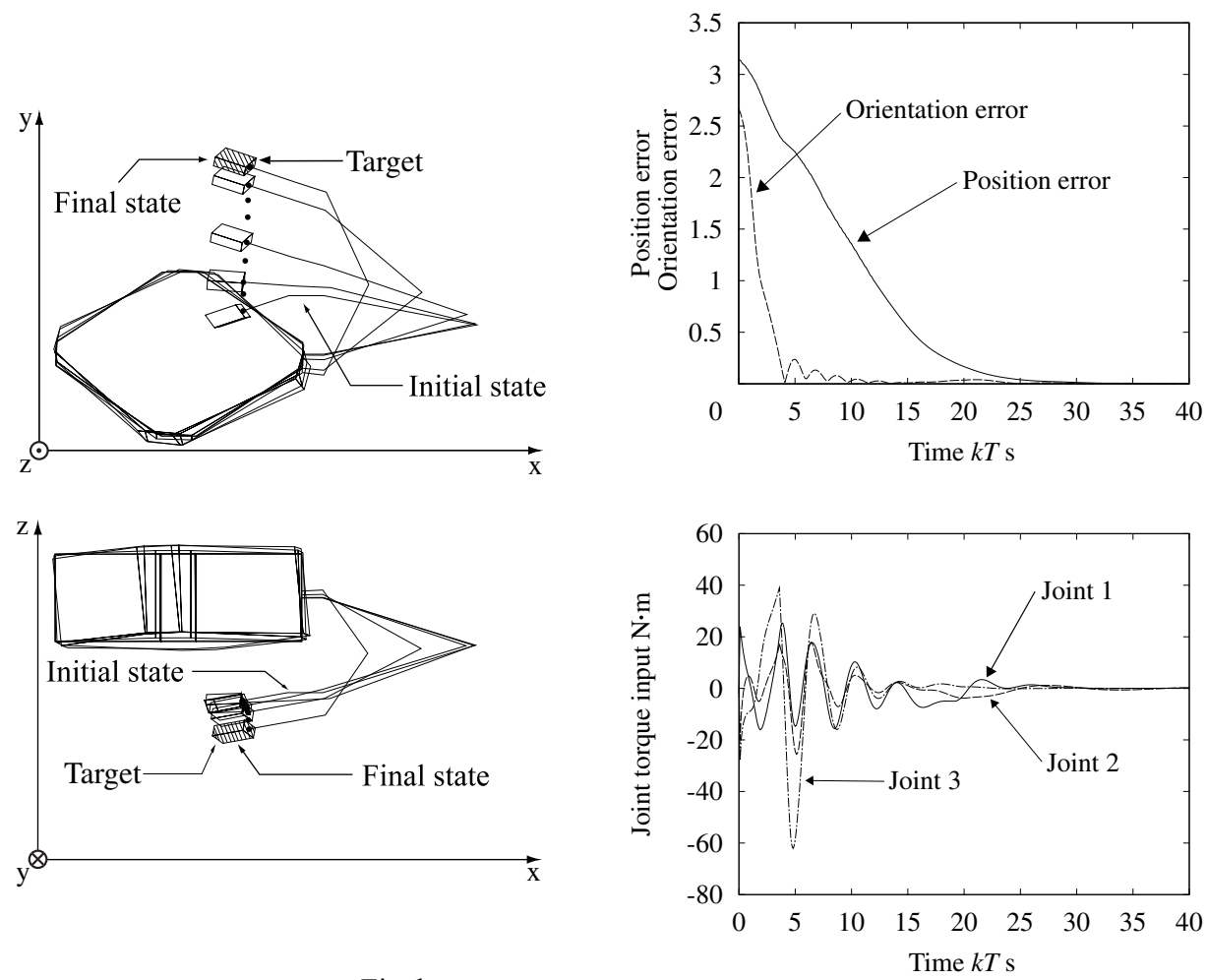

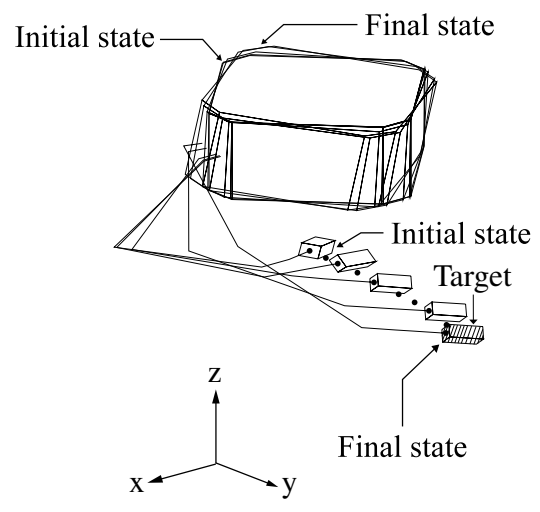

(a) Motion

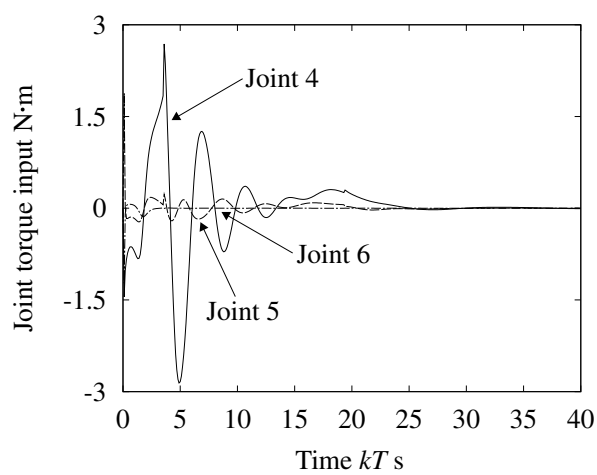

(b) Time history

Fig. 3. Simulation result of 6-DOF robot using basic control law (18) 


\subsection{Tracking control}

To apply the basic control law (18) to tracking control, the following equations are utilized instead of Equations (8) and (17):

$$
\begin{gathered}
\overline{\boldsymbol{e}}_{L}(k)=\boldsymbol{p}_{T}(k)-\boldsymbol{p}_{E}(k), \\
\overline{\boldsymbol{e}}_{A}(k)=-\frac{1}{2} \boldsymbol{E}_{X}^{T}(k) \overline{\boldsymbol{E}}_{A}(k)
\end{gathered}
$$

where

$$
\overline{\boldsymbol{E}}_{A}(k)=\left[\begin{array}{c}
\boldsymbol{n}_{T}(k)-\boldsymbol{n}_{E}(k) \\
\boldsymbol{s}_{T}(k)-\boldsymbol{s}_{E}(k) \\
\boldsymbol{a}_{T}(k)-\boldsymbol{a}_{E}(k)
\end{array}\right] .
$$

To examine the performance of the tracking control using Equation (18), three types of simulations were performed using a horizontal planar 3-DOF robot shown in Figure 4 with object.

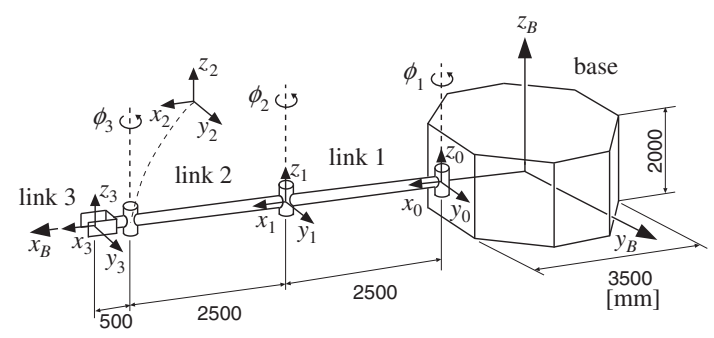

\begin{tabular}{|c|c|c|c|}
\hline & Length[m] & Mass $[\mathrm{kg}]$ & Moment of inertia $\left[\mathrm{kg} \cdot \mathrm{m}^{2}\right]$ \\
\hline Base & 3.5 & 2000 & 3587.9 \\
\hline Link 1 & 2.5 & 50 & 26.2 \\
\hline Link 2 & 2.5 & 50 & 26.2 \\
\hline Link 3 & 0.5 & 5 & 0.23 \\
\hline Object & 4.0 & 100 & 200.0 \\
\hline
\end{tabular}

Fig. 4. Simulation model of 3-DOF space robot

Simulations were carried out under the following condition. A point of interest of the object moves along a straight path from the initial position to the target position, and the object angle is set to the initial value. The sampling period is $T=0.01 \mathrm{~s}$ and the feedback gains are set for the following cases.

Case 1: This is the basic case. The feedback gains are $k_{L}=k_{A}=50000, K_{L}=$ $\operatorname{diag}\{5000,5000\}$ and $\boldsymbol{K}_{A}=5000$.

Case 2: Position and orientation feedback gains, $k_{L}$ and $k_{A}$, are set to larger values than those in Case 1. The gains are $k_{L}=k_{A}=100000, \boldsymbol{K}_{L}=\operatorname{diag}\{5000,5000\}$ and $\boldsymbol{K}_{A}=5000$.

Case 3: Linear and angular velocity feedback gains $\boldsymbol{K}_{L}$ and $\boldsymbol{K}_{A}$ are set to smaller values than those in Case 1. The gains are $k_{L}=k_{A}=50000, \boldsymbol{K}_{L}=\operatorname{diag}\{3000,3000\}$ and $\boldsymbol{K}_{A}=3000$. 


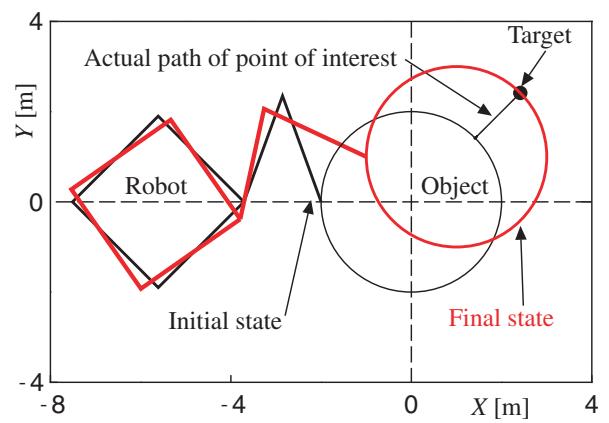

(a) Motion (Case 1)

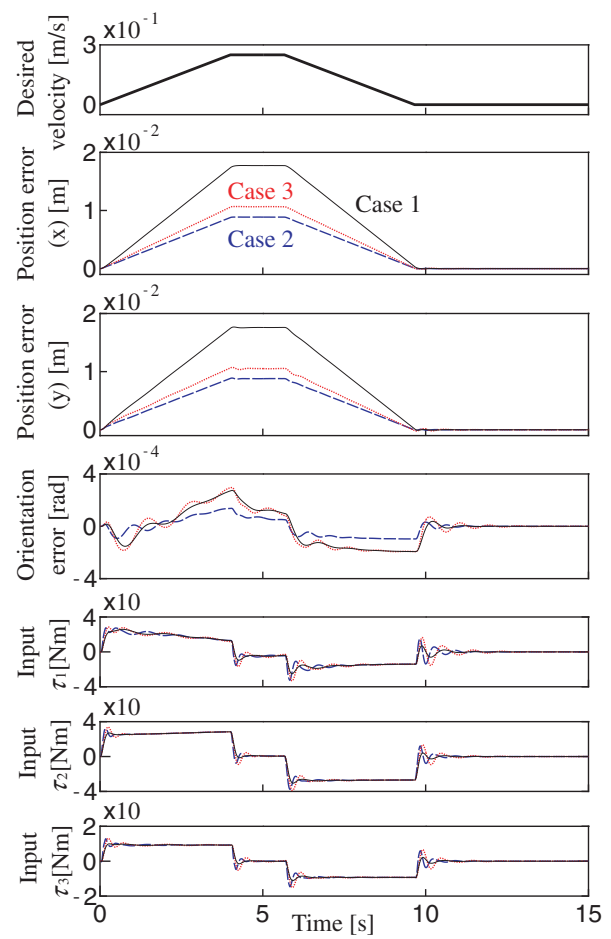

(b) Time history

Fig. 5. Simulation result of using basic control law (18) 
Figure 5(a) shows the motion of the robot in Case 1. From Figure 5(a), it can be seen that tracking control using the control law (18) is valid. Figure 5(b) shows the simulation results in all cases. This figure consists of the norm of the desired linear velocity of the object, the position errors of $x$ and $y$ directions, the orientation error, and the control inputs of joint 1 , 2 and 3. Furthermore, Figure 6 shows the time-history of the position and orientation errors after the object reaches the vicinity of the target. From Figure 5(b), the tracking errors in Case 2 and 3 are smaller than the errors in Case 1, while the desired velocity is transitional. From Figure 6, however, the convergent performance of the target becomes slightly worse.

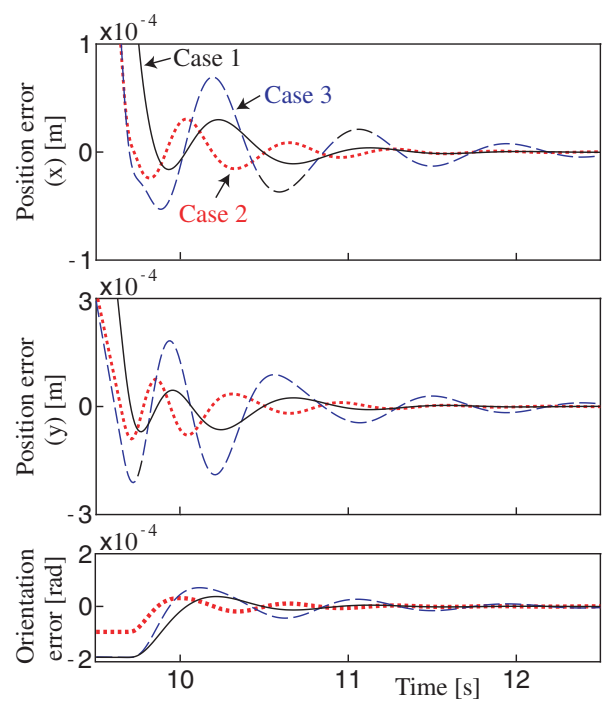

Fig. 6. Position and orientation errors after the object reaches the vicinity of the target

So, to get good control performance, we propose the modified control law (Sagara \& Taira, 2007):

$$
\boldsymbol{\tau}_{d}(k)=\boldsymbol{J}_{L}^{T}(k)\left\{\hat{k}_{L}(k) \hat{\boldsymbol{e}}_{L}(k)-\hat{\boldsymbol{K}}_{L}(k) \boldsymbol{v}_{E}(k)\right\}+\boldsymbol{J}_{A}^{T}(k)\left\{\hat{k}_{A}(k) \hat{\boldsymbol{e}}_{A}(k)-\hat{\boldsymbol{K}}_{A}(k) \boldsymbol{\omega}_{E}(k)\right\}
$$

where

$$
\begin{gathered}
\hat{k}_{\boldsymbol{+}}(k)=k_{\boldsymbol{+}}\left\{1+\alpha_{+} v_{+}(k)\right\}, \quad \hat{\boldsymbol{K}}_{\boldsymbol{+}}(k)=\boldsymbol{K}_{+}\left\{1-\beta_{+} v_{+}(k)\right\} \quad(\boldsymbol{\dagger}=L, A), \\
v_{L}(k)=\frac{\left\|\boldsymbol{v}_{\text {int }_{d}}(k)\right\|}{v_{d_{\max }}}, \quad v_{A}(k)=\frac{\left\|\boldsymbol{\omega}_{\text {int }_{d}}(k)\right\|}{\omega_{d_{\max }}},
\end{gathered}
$$

and $\boldsymbol{v}_{E_{d}}(k)$ and $\boldsymbol{\omega}_{E_{d}}(k)$ are the desired velocities of $\boldsymbol{v}_{E}(k)$ and $\boldsymbol{\omega}_{E}(k)$, respectively, $v_{d_{\max }}$ and $\omega_{d_{\max }}$ are the maximum values of the norm of $\boldsymbol{v}_{E_{d}}(k)$ and $\boldsymbol{\omega}_{E_{d}}(k), \alpha_{*}\left(\alpha_{*} \geq 0\right)$ and $\beta_{*}(0 \leq$ $\left.\beta_{*} \leq 1\right)(*=L, A)$ are setting parameters.

To verify the validity of the tracking control law (38), a simulation was performed. The values of the feedback gains, $k_{L}, k_{A}, \boldsymbol{K}_{L}$ and $\boldsymbol{K}_{A}$, for this simulation and for Case 1 are the same. The setting parameters are $\alpha_{A}=\alpha_{L}=0.8$ and $\beta_{A}=\beta_{L}=0.3$. 

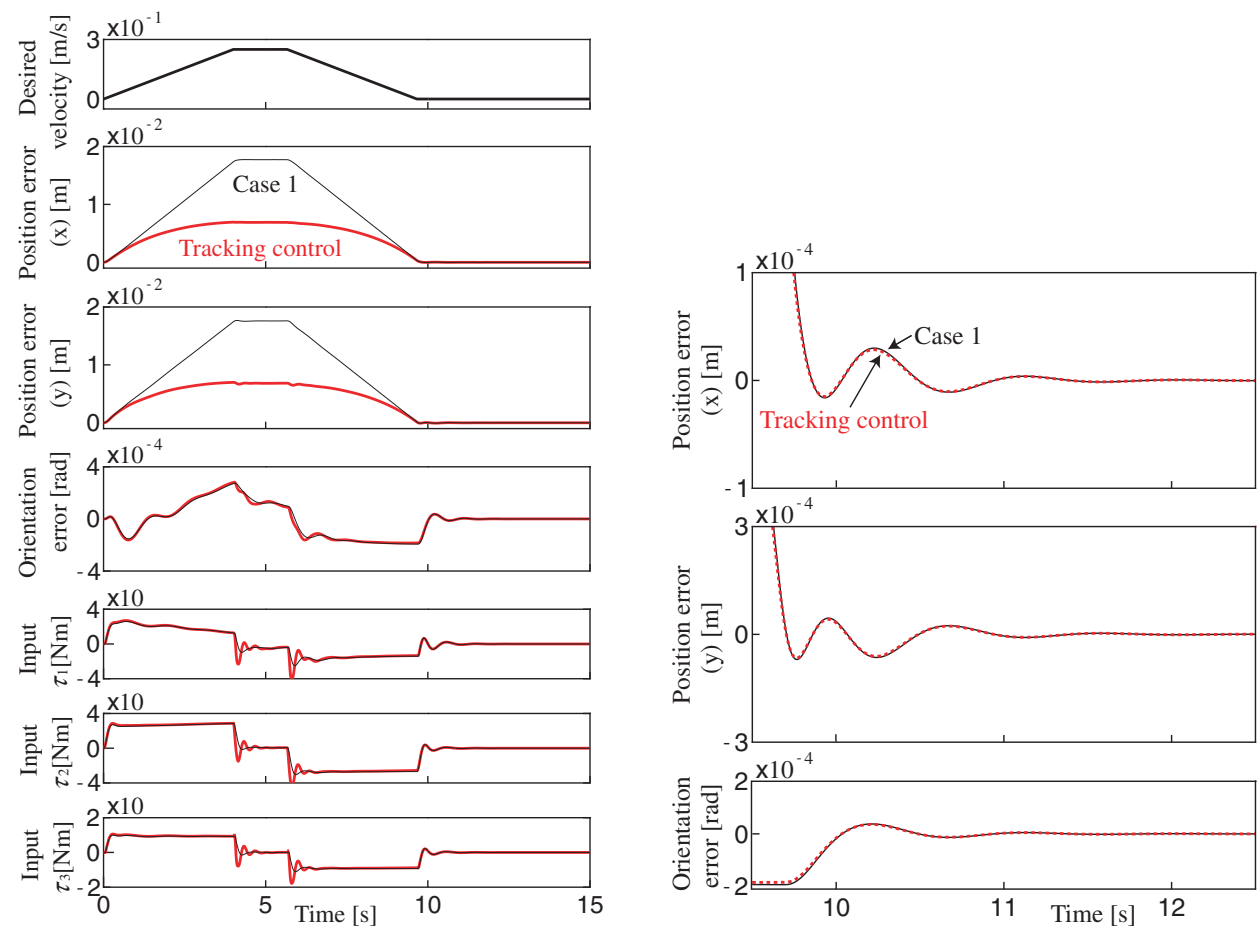

Fig. 7. Simulation result using tracking control law (38)

Figure 7 shows simulation result using the tracking control law (38). From Figure 7, it can be seen that good control performance can be achieved using the tracking control law.

\subsection{Tracking control for joint velocity controller}

The tracking control law (38) can be utilized for manipulators equipped with joint torque controller. It is considered that joint velocity controllers are also used for space robot manipulators. In this subsection, we address a tracking control method for robot manipulators equipped with joint velocity controller (Sagara \& Taira, 2008b).

Figure 8(a) shows the time-history of the joint torque and velocity of the simulation result in case of Figure 7. And Figure 8(b) shows the relation between the actual joint input torque and the joint angular velocity. From Figure 8(b) we can see that the value of angular velocity is varying with the constant torque input during the sampling interval $T$. In other words, if the control inputs vary roughly for manipulators with joint velocity controllers, the joint controllers give large torques to the robot.

For manipulators with joint velocity controllers the tracking control law (38) cannot be applied directly. To obtain similar control performance to the case of the joint torque controllers, we use the following discrete-time dynamic equation of the robot.

$$
\dot{\phi}\left(k_{1}\right)=\dot{\phi}\left(k_{1}-1\right)-T_{1} \boldsymbol{H}^{-1}\left(k_{1}\right)\left\{\boldsymbol{c}\left(k_{1}\right)-\boldsymbol{\tau}\left(k_{1}\right)\right\}
$$




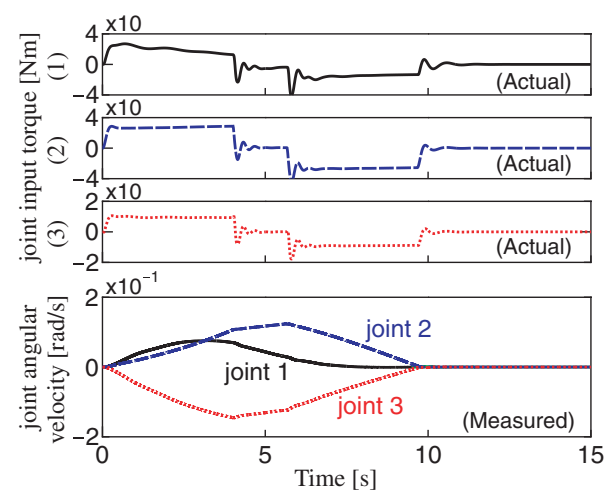

(a) All joints

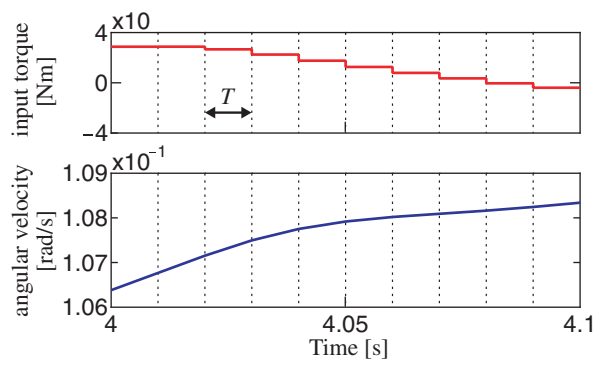

(b) Relation between torque and velocity of joint 2

Fig. 8. Joint torque and velocity in case of tracking control for joint torque controller

where the sampling period $T_{1}$ ( $T=n T_{1}, n$ is positive integer) is used to Equation (7) instead of $T$, and $\boldsymbol{c}\left(k_{1}\right)=\boldsymbol{C}\left(k_{1}\right) \dot{\boldsymbol{\phi}}\left(k_{1}\right)$. Here, we assume that $\boldsymbol{\phi}$ is constant during the sampling interval $T$ and $\boldsymbol{c}\left(k_{1}\right) \approx \boldsymbol{c}\left(k_{1}-1\right)$ during the sampling interval $T_{1}$. Then for Equation (39) the actual joint velocity control input $\dot{\phi}_{d}\left(k_{1}\right)$ is determined as

$$
\dot{\phi}_{d}\left(k_{1}\right)=\dot{\phi}\left(k_{1}-1\right)-T_{1} \boldsymbol{H}^{-1}(k)\left\{\boldsymbol{c}\left(k_{1}-1\right)-\boldsymbol{\tau}(k)\right\}
$$

To verify the validity of the control law (38) with Equation (40) simulations were performed. The condition is the same to the torque input case and the sampling period for Eq. (40) is $T_{1}=0.001 \mathrm{~s}(n=10)$.

Simulation result is shown in Figure 9. Furthermore, Figure 10 shows the difference of the joint angular velocity between the case of torque input control and velocity input control. From these figures, the both control performances are similar and good control performance can be achieved using the control law (38) with Equation (40).

\section{Cooperative manipulation of object}

It is considered that many tasks will be achieved by cooperative motions of some space robots in future space missions. We have studied on control problems for realizing cooperative manipulations and reported that a system consisting of some space robots with manipulators and a floating object can be treated as a kind of distributed system (Katoh et al., 1997; Sagara et al., 1998b). Using the distributed system representation each robot constituting the distributed system can be designed the control system individually.

In this section, the tracking control method using the transpose of the GJM is applied to cooperative manipulations of a floating object by some space robots (Sagara \& Taira, 2008a; 2009).

\subsection{Robot system model}

In this subsection, we consider a space robot system consisting of $M$ robots with manipulators and a floating object shown in Figure 11. The $h$-th robot $(h=1, \cdots, M)$ is consisting of an uncontrolled base and $n_{h}$-DOF manipulator with revolute joints. 


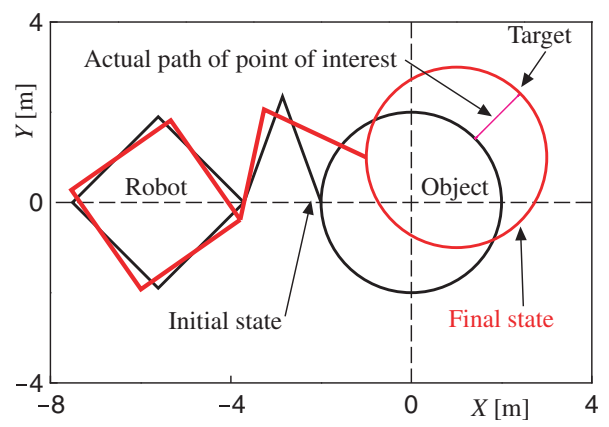

(a) Motion
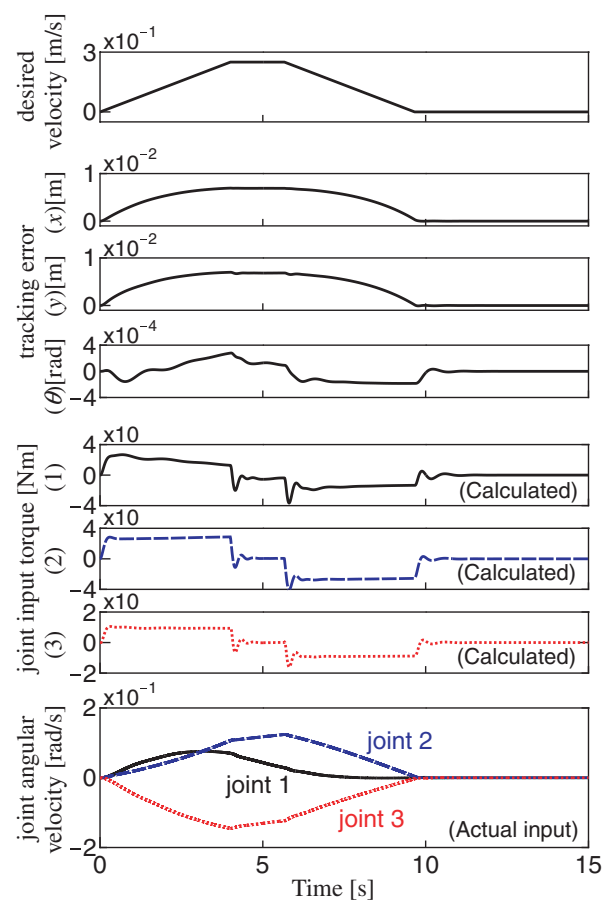

(b) Time history

Fig. 9. Simulation result using tracking control for joint velocity controller 


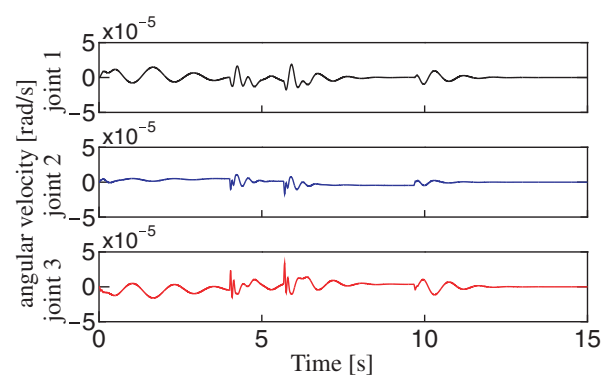

Fig. 10. Error comparison between torque controller and velocity controller

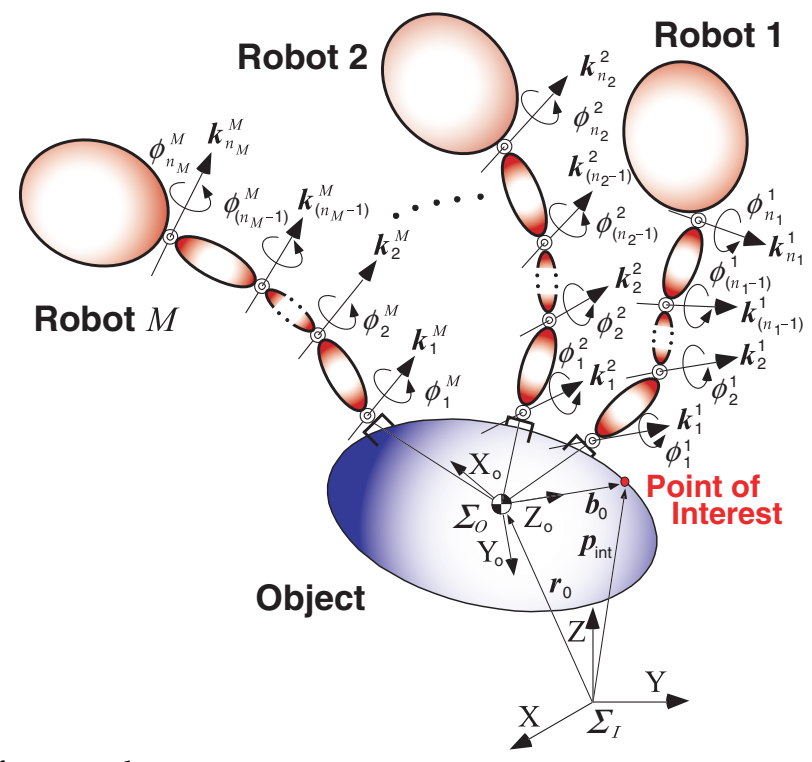

Fig. 11. Model of space robot system

The robot system shown in Figure 11 can be understood as one robot with $M$ manipulators by regarding the object as a robot body, and $M$ robot arms and robot bodies as $M$ manipulators. The kinematic formulation of such space system has been derived by (Yoshida et al., 1991a;b). So, several symbols defined in Section 2 are changed. These symbols used in this section are redefined as follows:

$r_{0}:$ position vector of center of mass of base

$\boldsymbol{p}_{E}:$ position vector of center of object

$p_{\text {int }}:$ position vector of point of interest on object

$\boldsymbol{p}_{T}$ : position vector of point of interest

$\boldsymbol{v}_{0}$ : linear velocity vector of center of mass of object

$v_{\text {int }}$ : linear velocity vector of point of interest 
$\boldsymbol{\omega}_{0}:$ angular velocity vector of center of mass of object

$\omega_{\text {int }}$ : angular velocity vector of point of interest

$i^{h}$ : number of link or joint $i$ of robot $h$

$\boldsymbol{p}_{i}^{h}$ : position vector of joint $i^{h}$

$\boldsymbol{r}_{i}^{h}$ : position vector of mass center of link $i^{h}$

$\boldsymbol{k}_{i}^{h}:$ unit vector indicating joint axis direction of joint $i^{h}$

$\boldsymbol{r}_{g}^{h}:$ position vector of mass center of robot $h$

$\phi_{i}^{h}:$ relative angle of joint $i^{h}$

$m_{0}:$ mass of object

$m_{i}^{h}:$ mass of link $i^{h}$

$\boldsymbol{I}_{0}$ : inertia tensor of object

$\boldsymbol{I}_{i}^{h}$ : inertia tensor of link $i^{h}$

The relation obtained from the geometrical relationships of the robot system, and the conservation laws of linear momentum and angular momentum as follows:

$$
\boldsymbol{\nu}_{\mathrm{int}}=\left[\begin{array}{c}
\boldsymbol{v}_{\mathrm{int}} \\
\boldsymbol{\omega}_{\mathrm{int}}
\end{array}\right]=\boldsymbol{J}_{s}^{c}\left[\begin{array}{c}
\boldsymbol{v}_{0} \\
\boldsymbol{\omega}_{0}
\end{array}\right], \quad \boldsymbol{H}_{s}^{c}\left[\begin{array}{c}
\boldsymbol{v}_{0} \\
\boldsymbol{\omega}_{0}
\end{array}\right]+\boldsymbol{H}_{m}^{c} \dot{\boldsymbol{\phi}}=\mathbf{0}
$$

where

$$
\begin{aligned}
& \boldsymbol{J}_{s}^{c}=\left[\begin{array}{cc}
\boldsymbol{E} & \tilde{\boldsymbol{r}}_{0}-\tilde{\boldsymbol{p}}_{\mathrm{int}} \\
\mathbf{0} & \boldsymbol{E}
\end{array}\right], \quad \boldsymbol{H}_{s}^{c}=\left[\begin{array}{cc}
w \boldsymbol{E} & w\left(\tilde{\boldsymbol{r}}_{0}-\tilde{\boldsymbol{r}}_{g}\right) \\
w \tilde{\boldsymbol{r}}_{g} & \boldsymbol{I}_{w}^{c}
\end{array}\right], \quad \boldsymbol{H}_{m}^{c}=\left[\begin{array}{c}
\boldsymbol{J}_{T_{w}}^{c} \\
\boldsymbol{I}_{\phi}^{c}
\end{array}\right], \\
& \phi^{c}=\left[\left(\phi^{1}\right)^{T},\left(\phi^{2}\right)^{T}, \cdots,\left(\phi^{M}\right)^{T}\right]^{T}, \\
& \boldsymbol{I}_{w}^{c}=\sum_{h=1}^{M} \boldsymbol{I}_{w}^{h}+\boldsymbol{I}_{o}, \quad \boldsymbol{J}_{T_{w}}^{c}=\sum_{h=1}^{M} \boldsymbol{J}_{T_{w}}^{h}, \quad \boldsymbol{I}_{\phi}^{c}=\sum_{h=1}^{M} \boldsymbol{I}_{\phi}^{h} \\
& \boldsymbol{I}_{w}^{h}=\sum_{i=1}^{n_{h}}\left\{\boldsymbol{I}_{i}^{h}-m_{i}^{h} \tilde{\boldsymbol{r}}_{i}^{h}\left(\tilde{\boldsymbol{r}}_{i}^{h}-\tilde{\boldsymbol{r}}_{0}^{h}\right)\right\}, \quad \boldsymbol{J}_{T_{w}}^{h}=\sum_{i=1}^{n_{h}} m_{i}^{h} \boldsymbol{J}_{T_{i}}^{h}, \quad \boldsymbol{I}_{\phi}^{h}=\sum_{i=1}^{n_{h}}\left(\boldsymbol{I}_{i}^{h} \boldsymbol{J}_{R_{i}}^{h}+m_{i}^{h} \tilde{\boldsymbol{r}}_{i}^{h} \boldsymbol{J}_{T_{i}}^{h}\right), \\
& \boldsymbol{J}_{T_{i}}^{h}=\left[\begin{array}{lll}
\boldsymbol{O}_{a} & \overline{\boldsymbol{J}}_{T_{i}}^{h} & \boldsymbol{O}_{b}
\end{array}\right], \quad \boldsymbol{J}_{R_{i}}^{h}=\left[\begin{array}{lll}
\boldsymbol{O}_{a} & \overline{\boldsymbol{J}}_{R_{i}}^{h} & \boldsymbol{O}_{b}
\end{array}\right], \\
& \overline{\boldsymbol{J}}_{T_{i}}^{h}=\left[\tilde{\boldsymbol{k}}_{1}^{h}\left(\boldsymbol{r}_{i}^{h}-\boldsymbol{p}_{1}^{h}\right), \cdots, \tilde{\boldsymbol{k}}_{i}^{h}\left(\boldsymbol{r}_{i}^{h}-\boldsymbol{p}_{i}^{h}\right), \mathbf{0}, \cdots, \mathbf{0}\right], \quad \overline{\boldsymbol{J}}_{R_{i}}^{h}=\left[\boldsymbol{k}_{1}^{h}, \cdots, \boldsymbol{k}_{i}^{h}, \mathbf{0}, \cdots, \mathbf{0}\right],
\end{aligned}
$$

and $\boldsymbol{O}_{a} \in \boldsymbol{R}^{3 \times n_{a}}\left(n_{a}=\sum_{i=1}^{h-1} n_{i}\right)$ and $\boldsymbol{O}_{b} \in \boldsymbol{R}^{3 \times n_{b}}\left(n_{b}=\sum_{i=h+1}^{M} n_{i}\right)$ are zero matrices.

Form Equation (41), the relation between velocity $\nu_{\text {int }}$ of the object and joint angular velocity $\dot{\phi}^{c}$ of the manipulator can be derived as

$$
\nu_{\text {int }}=\boldsymbol{J}^{c} \dot{\phi}^{c}
$$

where $\boldsymbol{J}^{c}=-\boldsymbol{J}_{S}^{c}\left(\boldsymbol{H}_{s}^{c}\right)^{-1} \boldsymbol{H}_{m}^{c}$ is a GJM of the system shown in Figure 11. 


\subsection{System partition}

For the system shown in Figure 11 control systems can be easily constructed by using Equation (42). However, if the number of robots is changed, Equation (42) must be recalculated. Furthermore, if the number of robots becomes increased, a large amount of calculation for the system is necessary. To solve the problems described above, This total robot system is regarded as a distributed system.

By examining parameters and variables included in the matrix $\boldsymbol{H}_{s}^{c}$ and vector $\boldsymbol{H}_{m}^{c} \dot{\phi}^{c}$ in Equation (41), the matrix and vector can be rewritten as

$$
\boldsymbol{H}_{s}^{c}=\boldsymbol{H}_{s}^{0}+\sum_{h=1}^{M} \boldsymbol{H}_{s}^{h}, \quad \boldsymbol{H}_{m}^{c} \dot{\boldsymbol{\phi}}^{c}=\sum_{h=1}^{M} \boldsymbol{H}_{m}^{h} \dot{\boldsymbol{\phi}}^{h}
$$

where

$$
\begin{gathered}
\boldsymbol{H}_{s}^{0}=\left[\begin{array}{cc}
m_{0} \boldsymbol{E} & \mathbf{0} \\
m_{0} \tilde{\boldsymbol{r}}_{0} & \boldsymbol{I}_{0}
\end{array}\right], \quad \boldsymbol{H}_{s}^{h}=\left[\begin{array}{cc}
m^{h} \boldsymbol{E} & m^{h}\left(\tilde{\boldsymbol{r}}_{0}^{h}-\tilde{\boldsymbol{r}}_{g}^{h}\right) \\
m^{h} \tilde{\boldsymbol{r}}_{g}^{h} & \boldsymbol{I}_{w}^{h}
\end{array}\right], \quad \boldsymbol{H}_{m}^{h}=\left[\begin{array}{c}
\overline{\boldsymbol{J}}_{T_{w}}^{h} \\
\overline{\boldsymbol{I}}_{\phi}^{h}
\end{array}\right], \\
m^{h}=\sum_{i=1}^{n_{h}} m_{i}^{h}, \quad \overline{\boldsymbol{J}}_{T_{w}}^{h}=\sum_{i=1}^{n_{h}} m_{i}^{h} \overline{\boldsymbol{J}}_{T_{i}}^{h}, \quad \overline{\boldsymbol{I}}_{\phi}^{h}=\sum_{i=1}^{n_{h}}\left(\boldsymbol{I}_{i}^{h} \overline{\boldsymbol{J}}_{R_{i}}^{h}+m_{i}^{h} \tilde{\boldsymbol{r}}_{i}^{h} \overline{\boldsymbol{J}}_{T_{i}}^{h}\right) .
\end{gathered}
$$

$\boldsymbol{H}_{s}^{h}$ and $\boldsymbol{H}_{m}^{h}$ are matrices including parameters the $h$-th robot only, and $\boldsymbol{H}_{s}^{0}$ is a matrix including parameters of the object only.

Equations (41) and (43) make the following relation:

$$
\left(\boldsymbol{H}_{S}^{0}+\sum_{h=1}^{M} \boldsymbol{H}_{s}^{h}\right)\left(\boldsymbol{J}_{S}^{c}\right)^{-1} \boldsymbol{\nu}_{\mathrm{int}}+\sum_{h=1}^{M} \boldsymbol{H}_{m}^{h} \dot{\boldsymbol{\phi}}^{h}=\mathbf{0} .
$$

It is clear that the following set of equations is one of solutions of Equation (44), when a constant and diagonal matrix $\boldsymbol{A}_{h}$ is introduced.

$$
\overline{\boldsymbol{H}}_{s}^{h}\left(\boldsymbol{J}_{s}^{c}\right)^{-1} \boldsymbol{\nu}_{\mathrm{int}}+\boldsymbol{H}_{m}^{h} \dot{\boldsymbol{\phi}}^{h}=\mathbf{0} \quad(h=1, \cdots, M)
$$

where

$$
\overline{\boldsymbol{H}}_{s}^{h}=\boldsymbol{H}_{s}^{h}+\boldsymbol{A}_{h} \boldsymbol{H}_{s}^{0}, \quad \sum_{h=1}^{M} \boldsymbol{A}_{h}=\boldsymbol{E} .
$$

Then, the following relation can be derived from Equations (45).

$$
\boldsymbol{\nu}_{\mathrm{int}}=-\boldsymbol{J}_{s}^{c}\left(\overline{\boldsymbol{H}}_{s}^{h}\right)^{-1} \boldsymbol{H}_{m}^{h} \dot{\boldsymbol{\phi}}^{h} \quad(h=1, \cdots, M) .
$$

Therefore, for each robot of the system the control system can be designed individually.

\subsection{Simulation}

To examine the performance of the control methods using the transpose of the GJM, simulations are performed by using three of the horizontal planar 3-DOF robots shown in Figure 12 and an object. Note that the physical parameters of the robots shown in Figure 12 and 4 are the same, except for numbering the links and the joints.

All simulations were carried out under the following condition. A point of interest on the object moves along a straight path from the initial position to the target position and the object 


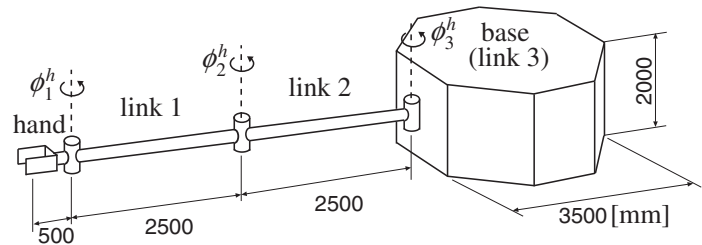

Fig. 12. Simulation model of 3-DOF space robot

angle is set up the initial value. The sampling period is $T=0.01 \mathrm{~s}$ and the coefficient matrices are $\boldsymbol{A}_{1}=\boldsymbol{A}_{2}=0.33 \boldsymbol{E}$ and $\boldsymbol{A}_{3}=0.34 \boldsymbol{E}$.

\subsubsection{Robustness for singular configuration}

Our proposed control methods using the transpose of the GJM can be utilized to the case of a singular configuration of manipulator. The robustness for the singular configuration is demonstrated by simulations using the basic control law (18).

Simulations were carried out under the following condition. The feedback gains are $k_{L}=$ $k_{A}=3 \times 10^{4}, \boldsymbol{K}_{L}=\operatorname{diag}\left\{2 \times 10^{3}, 2 \times 10^{3}\right\}$ and $\boldsymbol{K}_{A}=2 \times 10^{3}$. Furthermore, one of the robots breaks down $2.5 \mathrm{~s}$ after simulations are started, and the physical parameters of the floating object are shown in Table 2.

\begin{tabular}{|l|c|c|c|}
\hline & Diameter $[\mathrm{m}]$ & Mass $[\mathrm{kg}]$ & Moment of inertia $\left[\mathrm{kg} \cdot \mathrm{m}^{2}\right]$ \\
\hline Object & 3.0 & 100 & 106.2 \\
\hline
\end{tabular}

Table 2. Physical parameters of floating object

Figure 13 shows the simulation result. From this figure, we can see that the determinant of GJM of Robot 1 changes from positive values to negative values affected by the breakdown of Robot 3 , and the floating object can be manipulated by the robot system in spite of the singular configuration of Robot 1 . Therefore, our proposed control methods using the transpose of the GJM have the robustness for the singular configuration of manipulator.

\subsubsection{Tracking control for joint torque controller}

Next, the tracking control law (38) is applied to the robot system (Sagara \& Taira, 2008a).

Simulations were carried out under the following condition. The feedback gains are $k_{L}=$ $k_{A}=2 \times 10^{5}, \boldsymbol{K}_{L}=\operatorname{diag}\left\{2 \times 10^{4}, 2 \times 10^{4}\right\}$ and $\boldsymbol{K}_{A}=2 \times 10^{4}$. The setting parameters are $\alpha_{+}=2$ and $\beta_{\dagger}=0.2(\dagger=L, A)$. The physical parameters of the floating object are shown in Table 3.

\begin{tabular}{|l|c|c|c|}
\hline & Diameter $[\mathrm{m}]$ & Mass $[\mathrm{kg}]$ & Moment of inertia $\left[\mathrm{kg} \cdot \mathrm{m}^{2}\right]$ \\
\hline Object & 4.0 & 1200 & 2400.0 \\
\hline
\end{tabular}

Table 3. Physical parameters of floating object for tracking control method

Figure 14 shows the simulation result. Figure 14(b) also shows the case of the basic control, i. e., $\alpha_{+}=\beta_{+}=0$. From Figure 14, it can be seen that good control performance can be achieved using the tracking control law (38). 


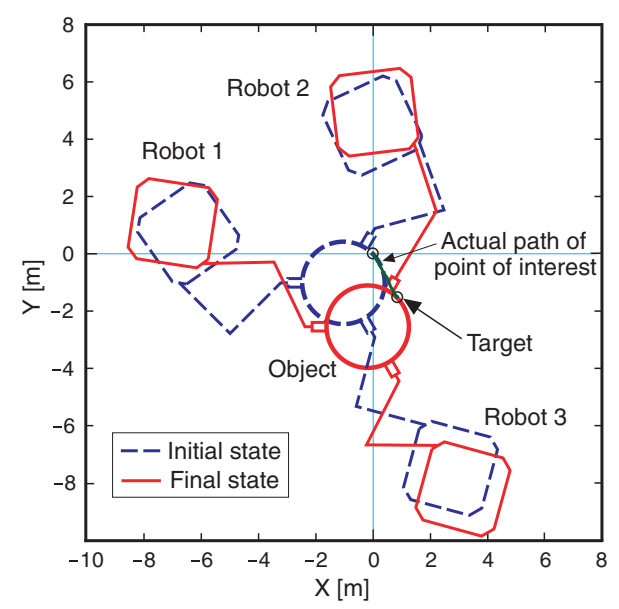

(a) Motion
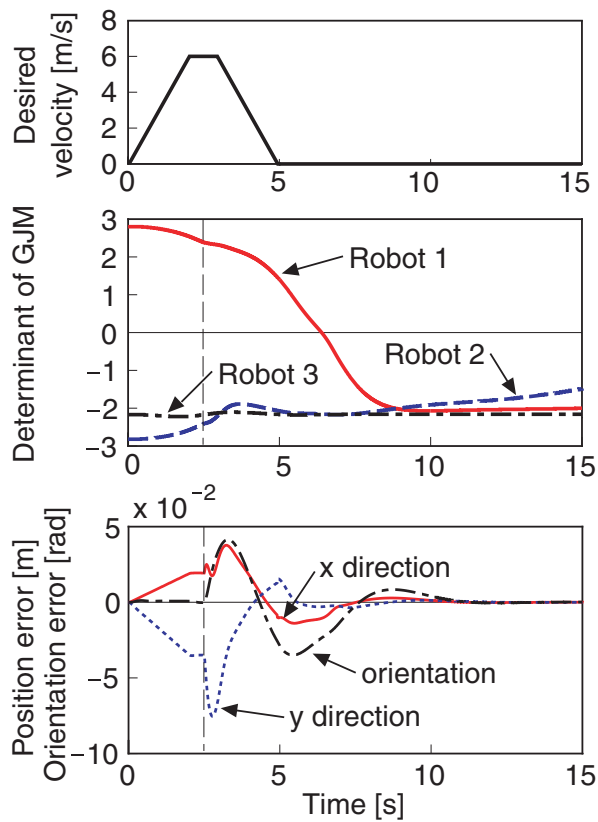

(b) Time history

Fig. 13. Simulation result of robot system using basic control law (18)

\subsubsection{Tracking control for joint velocity controller}

From the simulation result shown in Figure 9, the tracking control law (38) with discrete-time dynamic equation (40) can be applied to single manipulators equipped with joint velocity controllers. For the robot system shown in Figure 11, the dynamics of each robot is affected by other robots. Thus, the external force affected by other robots must be considered (Sagara \& Taira, 2009).

Based on the dynamic equation (39), the dynamic equation of the $h$-th robot shown in Figure 11 is obtained:

$$
\chi^{h}\left(k_{1}\right)=\chi^{h}\left(k_{1}-1\right)-T_{1} \boldsymbol{H}^{h^{-1}}\left(k_{1}\right)\left\{\boldsymbol{c}^{h}\left(k_{1}\right)-\boldsymbol{u}^{h}\left(k_{1}\right)\right\} .
$$

where

$$
\chi=\left[\begin{array}{c}
\eta_{0} \\
\dot{\phi}^{h}
\end{array}\right], \quad \boldsymbol{u}=\left[\begin{array}{l}
f^{h} \\
\tau^{h}
\end{array}\right],
$$

and $\boldsymbol{H}^{h}$ is the symmetric and positive definite inertia matrix, $\boldsymbol{c}^{h}$ is the vector of Coliolis and centrifugal forces, $\boldsymbol{\eta}_{0}=\left[\boldsymbol{v}_{0}{ }^{T}, \boldsymbol{\omega}_{0}{ }^{T}\right]^{T}$ is the velocity of the mass center of object, $\boldsymbol{f}^{h}$ is the external force affected by other robots.

For Eq. (47) the actual joint velocity control input $\dot{\phi}_{d}^{h}\left(k_{1}\right)$ is determined as

$$
\chi_{d}^{h}\left(k_{1}\right)=\chi^{h}\left(k_{1}-1\right)-T_{1} \boldsymbol{H}^{h^{-1}}(k)\left\{\boldsymbol{c}^{h}\left(k_{1}-1\right)-\boldsymbol{u}_{d}^{h}\left(k_{1}\right)\right\}
$$



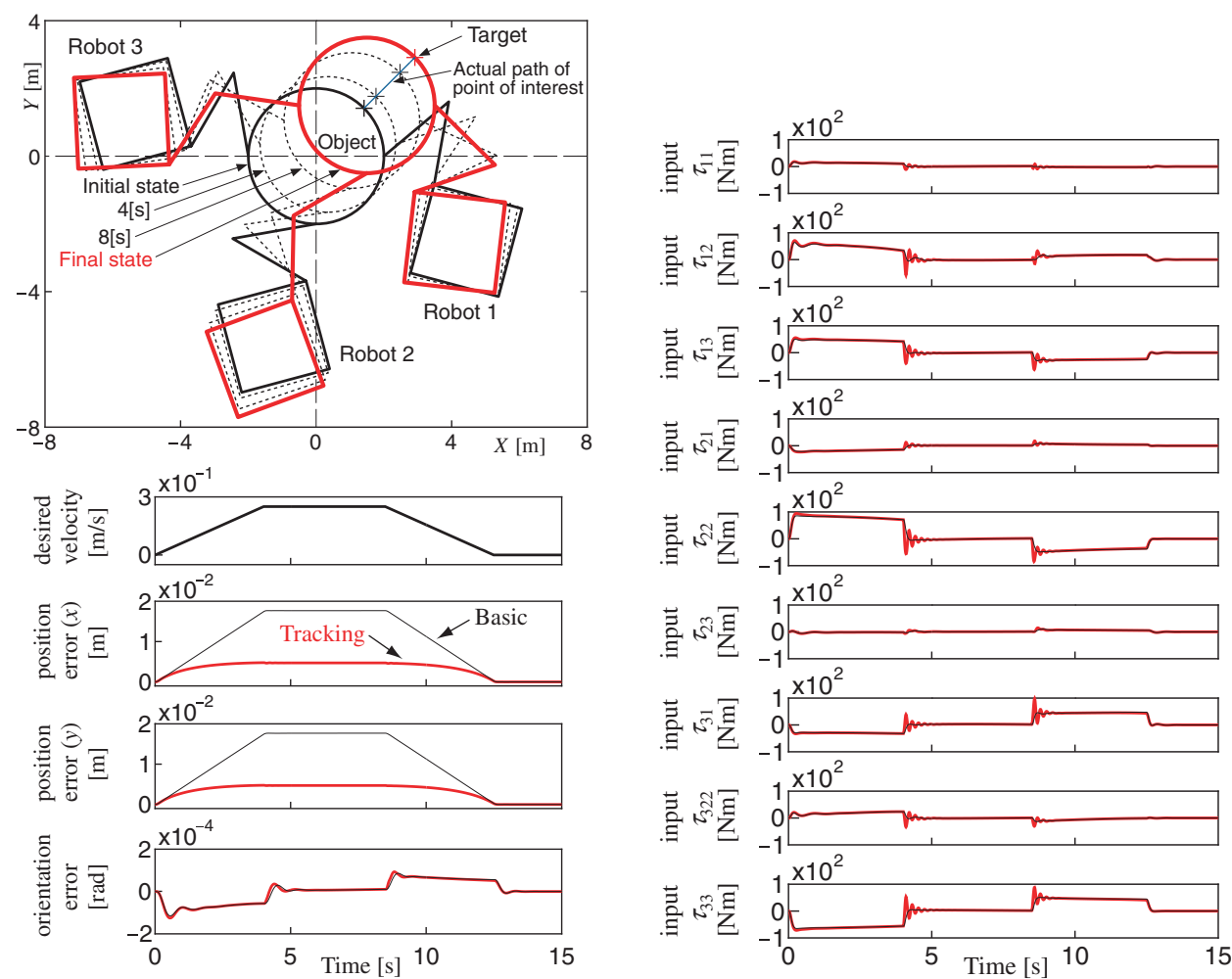

Fig. 14. Simulation result of robot system using tracking control law (38) (torque input)

where

$$
\boldsymbol{\chi}_{d}\left(k_{1}\right)=\left[\begin{array}{c}
\boldsymbol{\eta}_{0_{d}}^{h}\left(k_{1}\right) \\
\dot{\boldsymbol{\phi}}_{d}^{h}\left(k_{1}\right)
\end{array}\right], \quad \boldsymbol{u}_{d}\left(k_{1}\right)=\left[\begin{array}{c}
\boldsymbol{f}^{h}\left(k_{1}\right) \\
\boldsymbol{\tau}_{d}^{h}(k)
\end{array}\right] .
$$

Since the value of the external force $\boldsymbol{f}_{h}$ affected by other robots cannot obtained directly, a disturbance observer in discrete time (Godler et al., 2002) is used to estimate $\boldsymbol{f}_{h}$.

The equation of motion of the floating object with respect to the $h$-th robot is

$$
\boldsymbol{H}_{0}^{h}\left(k_{1}\right) \dot{\boldsymbol{\eta}}_{0}\left(k_{1}\right)=\boldsymbol{f}^{h}\left(k_{1}\right)+\overline{\boldsymbol{H}}_{0}^{h} \dot{\boldsymbol{\eta}}_{0_{d}}^{h}\left(k_{1}\right)
$$

where $\boldsymbol{H}_{0}^{h}\left(k_{1}\right)$ is the inertia matrix of the floating object and

$$
\overline{\boldsymbol{H}}_{0}^{h}=\boldsymbol{A}_{h}\left[\begin{array}{cc}
m_{0} \boldsymbol{E} & \mathbf{0} \\
\mathbf{0} & \boldsymbol{I}_{0}
\end{array}\right]
$$

is the nominal model of $\boldsymbol{H}_{0}^{h}\left(k_{1}\right)$.

For Equation (49) the estimated value of $\boldsymbol{f}_{h}, \hat{\boldsymbol{f}}_{h}$, can be obtained from the disturbance observer as shown in Figure 15. In this figure, (a), (b) and (c) show the basic configuration in 


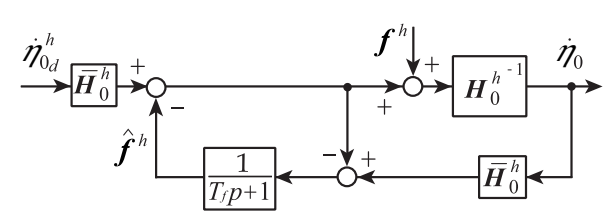

(a) Continuous-time

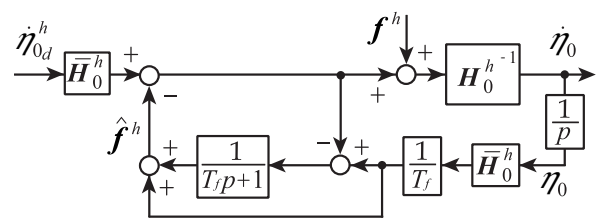

(b) Equivalent of (a)

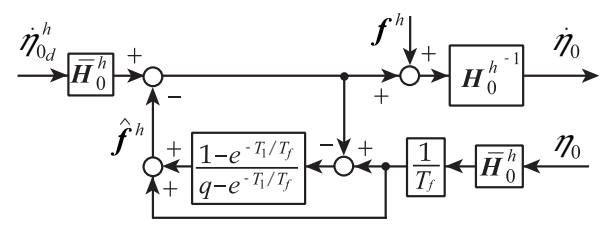

(c) Discrete-time

Fig. 15. Disturbance observer

continuous-time, the equivalent transformation of (a) and the discrete-time version, respectively, $T_{f}$ is a time constant of a low-pass filter, $p$ and $q$ are the differential and shift operators. From Figure 15(c), the estimated force can be obtained as follows:

$$
\begin{aligned}
\hat{\boldsymbol{f}}^{h}\left(k_{1}\right) & =\hat{\boldsymbol{f}}^{h}\left(k_{1}-1\right)+\left(1-e^{-T_{1} / T_{f}}\right) \overline{\boldsymbol{H}}_{0}^{h} \dot{\boldsymbol{\eta}}_{0_{d}}\left(k_{1}-1\right) \\
& +\frac{1}{T_{f}} \overline{\boldsymbol{H}}_{0}^{h}\left\{\boldsymbol{\eta}_{0}\left(k_{1}\right)+\left(1-2 e^{-T_{1} / T_{f}}\right) \boldsymbol{\eta}_{0}\left(k_{1}-1\right)\right\} .
\end{aligned}
$$

Simulations were performed to validate the effectiveness of the control method described above. The simulation condition for the joint velocity controllers is the same as those for the joint torque controllers. In addition, the time constant for the low-pass filter is $T_{f}=1 \mathrm{~s}$. Figure 16 shows the simulation result. From Figure 16(a), the object is successfully moved by three robots. And from Figure 16(b), it can be seen that good control performance can be achieved.

\section{Conclusion}

In this chapter, our proposed control methods for space robot manipulators using transpose of GJM were described and the computer simulations were performed. For manipulators equipped with joint torque controllers we explained about a basic control method using constant feedback gains with the proof of stability. To obtain higher control performance, we addressed trajectory tracking control methods with variable feedback gains for both torque and velocity joint inputs. Moreover, we addressed a cooperative manipulation of a floating object by some space robots with the control methods using transpose of GJM. For the cooperative manipulation, simulations where manipulators get into a singular configuration were also performed. 


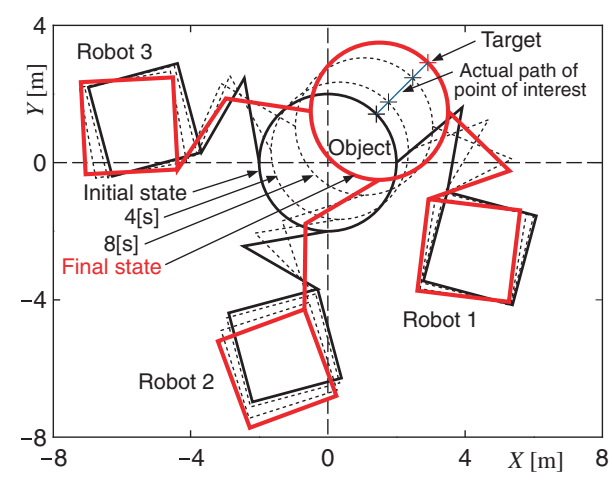

(a) Motion

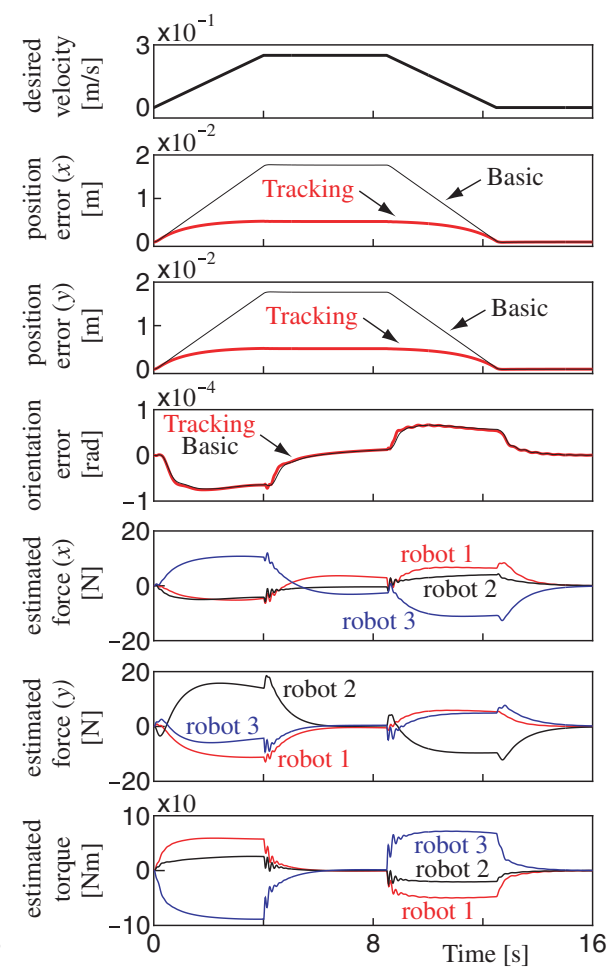

(b) Time history

Fig. 16. Simulation result of the robot system equipped with joint velocity controllers

\section{References}

Dubowsky, S. \& Papadorpulos, E. (1993). The Kinematics, Dynamics, and Control of FreeFlying and Free-Floating Space Robotic Systems, IEEE Transactions on Robotics and Automation, Vol. 9, No. 5, pp. 531-543

Godler, I., Honda, H. \& Ohnishi, K. (2002). Design Guidelines for Disturbance Observer's Filter in Discrete Time, Proceedings of the 7th International Workshop on Advanced Motion Control, Maribor, pp. $390-395$

Katoh, R., Nakatsuka, K., Sagara, S., Kobayshi, J. \& Ohkawa, F. (1997). Manipulation of a Floating Object by Two Space Manipulators, Proceedings of 23rd IEEE Industrial Electronics Society International Conference, pp. 1270-1275

Masutani, Y., Miyazaki, F. \& Arimoto, S. (1989a). Sensory Feedback Control for Space Manipulators, Proceedings 1989 IEEE International Conference on Robotics and Automation, pp. $1346-1351$

Masutani, Y., Miyazaki, F. \& Arimoto, S. (1989b). Modeling and Sensory Feedback Control for Space Manipulators, Proceedings of the NASA Conference on Space Telerobotics, pp. 287 $-296$ 
Sagara, S., Taira, Y., Katoh, R., Yamashita, T., Ohkawa, F. \& Suehiro, T. (1998a). Digital Adaptive Control of Space Robot Manipulator Having Input Constraints, Proceedings of the 1998 IEEE/RSJ International Conference on Intelligent Robots and Systems, pp. 1449-1454

Sagara, S., Hideura, M., Katoh, R., Yamashita, T., Kobayashi, J. \& Ohkawa, F. (1998b). Adaptive RMRC for Cooperative Manipulation of a Floating Object by Two Free-Based Space Robots, Proceedings of the 1998 IEEE/RSJ International Conference on Intelligent Robots and Systems, pp. 1467-1472

Sagara, S. \& Taira, Y. (2007). Digital Tracking Control of Space Robots Using a Transpose of the Generalized Jacobian Matrix, Artificial Life and Robotics, Vol. 11, pp. 82-86

Sagara, S. \& Taira, Y. (2008a). Cooperative Manipulation of a Floating Object by Some Space Robots: Application of a Tracking Control Method Using the Transpose of the Generalized Jacobian Matrix, Artificial Life and Robotics, Vol. 12, pp. 138-141

Sagara, S. \& Taira, Y. (2008b). Digital Control of Space Robot Manipulators with Velocity Type Joint Controller Using the Transpose of the Generalized Jacobian Matrix, Artificial Life and Robotics, Vol. 13, pp. 355-358

Sagara, S. \& Taira, Y. (2009). Cooperative Manipulation of a Floating Object by Some Space Robots with Joint Velocity Controllers: Application of a Tracking Control Method Using the Transpose of the Generalized Jacobian Matrix, Artificial Life and Robotics, Vol. 14, pp. 392-396

Shin, J. H., Jeong, I. K., Lee, J. J. \& Ham, W. (1995). Adaptive Robust Control for Free-Flying Space Robots Using Norm-Bounded Property of Uncertainty, Proceedings of the 1995 IEEE/RSJ International Conference on Intelligent Robots and Systems, pp. 59-64

Taira, Y., Sagara, S. \& Katoh, R. (2001). Digital Control of a Space Robot Manipulators Using Transpose of the Generalized Jacobian Matrix (in Japanese), Transactions of The Japan Society of Mechanical Engineers, Series C, Vol. 67, No. 663, pp. 3540-3547

Umetani, Y. \& Yoshida, K. (1989). Resolved Motion Rate Control of Space Manipulators with Generalized Jacobian Matrix, IEEE Transactions on Robotics and Automation, Vol. 5, No. 3, pp. $303-314$

Xu, Y. \& Kanade, T. (Ed.) (1993). Space Robotics: Dynamics and Control, Kluwer Academic Publishers, 0-7923-9265-5, Massachusetts

Yamamoto, T., Kobayashi, J., Katoh, R. \& Ohkawa, F. (1995). Digital Adaptive Control of a Manipulator Mounted on Free-Flying Robot in Space, Proceedings Ninth World Congress on the Theory of Machines and Mechanism, pp. 2151-2155

Yoshida, K., Kurazume, R \& Umetani, Y. (1991a). Dual Arm Coordination in Space Free-Flying Robot, Proceedings of the 1991 IEEE International Conference on Robotics and Automation, pp. 2516-2521

Yoshida, K., Kurazume, R \& Umetani, Y. (1991b). Coordinated control of multiple manipulators in space robots (in Japanese), Journal of the Robotics Society of Japan, Vol. 9, pp. 718-726 


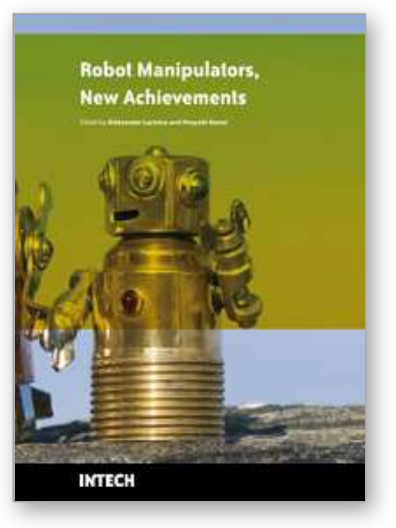

\author{
Robot Manipulators New Achievements \\ Edited by Aleksandar Lazinica and Hiroyuki Kawai
}

ISBN 978-953-307-090-2

Hard cover, 718 pages

Publisher InTech

Published online 01, April, 2010

Published in print edition April, 2010

Robot manipulators are developing more in the direction of industrial robots than of human workers. Recently, the applications of robot manipulators are spreading their focus, for example Da Vinci as a medical robot, ASIMO as a humanoid robot and so on. There are many research topics within the field of robot manipulators, e.g. motion planning, cooperation with a human, and fusion with external sensors like vision, haptic and force, etc. Moreover, these include both technical problems in the industry and theoretical problems in the academic fields. This book is a collection of papers presenting the latest research issues from around the world.

\title{
How to reference
}

In order to correctly reference this scholarly work, feel free to copy and paste the following:

Shinichi Sagara and Yuichiro Taira (2010). Digital Control of Free Floating Space Robot Manipulators Using Transpose of Generalized Jacobian Matrix, Robot Manipulators New Achievements, Aleksandar Lazinica and Hiroyuki Kawai (Ed.), ISBN: 978-953-307-090-2, InTech, Available from:

http://www.intechopen.com/books/robot-manipulators-new-achievements/digital-control-of-free-floating-spacerobot-manipulators-using-transpose-of-generalized-jacobian-ma

\section{INTECH}

open science | open minds

\section{InTech Europe}

University Campus STeP Ri

Slavka Krautzeka 83/A

51000 Rijeka, Croatia

Phone: +385 (51) 770447

Fax: +385 (51) 686166

www.intechopen.com

\section{InTech China}

Unit 405, Office Block, Hotel Equatorial Shanghai

No.65, Yan An Road (West), Shanghai, 200040, China

中国上海市延安西路65号上海国际贵都大饭店办公楼405单元

Phone: +86-21-62489820

Fax: $+86-21-62489821$ 
(C) 2010 The Author(s). Licensee IntechOpen. This chapter is distributed under the terms of the Creative Commons Attribution-NonCommercialShareAlike-3.0 License, which permits use, distribution and reproduction for non-commercial purposes, provided the original is properly cited and derivative works building on this content are distributed under the same license. 